Old Dominion University

ODU Digital Commons

2020

\title{
Extreme Ultraviolet Quasar Colours from GALEX Observations of the SDSS DR14Q Catalogue
}

\author{
Daniel E. Vanden Berk \\ Sarah C. Wesolowski \\ Mary J. Yeckley \\ Joseph M. Marcinik \\ Jean M. Quashnock
}

See next page for additional authors

Follow this and additional works at: https://digitalcommons.odu.edu/computerscience_fac_pubs

Part of the Computer Sciences Commons, and the External Galaxies Commons

\section{Original Publication Citation \\ Vanden Berk, D. E., Wesolowski, S. C., Yeckley, M. J., Marcinik, J. M., Quashnock, J. M., Machia, L. M., \& Wu, J. (2020). Extreme ultraviolet quasar colours from GALEX observations of the SDSS DR14Q catalogue. Monthly Notices of the Royal Astronomical Society, 493(2), 2745-2764. doi:10.1093/mnras/ staa411}

This Article is brought to you for free and open access by the Computer Science at ODU Digital Commons. It has been accepted for inclusion in Computer Science Faculty Publications by an authorized administrator of ODU Digital Commons. For more information, please contact digitalcommons@odu.edu. 


\section{Authors}

Daniel E. Vanden Berk, Sarah C. Wesolowski, Mary J. Yeckley, Joseph M. Marcinik, Jean M. Quashnock, Lawrence M. Machia, and Jian Wu 


\title{
Extreme ultraviolet quasar colours from GALEX observations of the SDSS DR14Q catalogue
}

\author{
Daniel E. Vanden Berk ${ }^{\oplus}{ }^{1 \star}$ Sarah C. Wesolowski, ${ }^{1,2,3}$ Mary J. Yeckley, ${ }^{1,4}$ \\ Joseph M. Marcinik, ${ }^{1,5}$ Jean M. Quashnock, ${ }^{6}$ Lawrence M. Machia ${ }^{1}$ and Jian $\mathrm{Wu}^{7}$ \\ ${ }^{1}$ Physics Department, Saint Vincent College, 300 Fraser Purchase Road, Latrobe, PA 15650, USA \\ ${ }^{2}$ Department of Physics, The Ohio State University, 191 West Woodruff Ave., Columbus, OH 43210, USA \\ ${ }^{3}$ Department of Mathematics and Computer Science, Salisbury University, 1101 Camden Ave., Salisbury, MD 21801, USA \\ ${ }^{4}$ Department of Physics and Astronomy, University of Maine, 5709 Bennett Hall, Orono, ME 04469, USA \\ ${ }^{5}$ Department of Physics and Astronomy, University of California, Los Angeles, 475 Portola Plaza, Los Angeles, CA 90095, USA \\ ${ }^{6}$ Department of Physics and Astronomy, Carthage College, 2001 Alford Park Drive, Kenosha, WI 53140, USA \\ ${ }^{7}$ Computer Science Department, Old Dominion University, Norfolk, VA 23529, USA
}

Accepted 2020 February 6. Received 2020 February 4; in original form 2019 November 1

\begin{abstract}
The rest-frame far to extreme ultraviolet (UV) colour-redshift relationship has been constructed from data on over 480000 quasars carefully cross-matched between SDSS Data Release 14 and the final GALEX photometric catalogue. UV matching and detection probabilities are given for all the quasars, including dependencies on separation, optical brightness, and redshift. Detection limits are also provided for all objects. The UV colour distributions are skewed redward at virtually all redshifts, especially when detection limits are accounted for. The median GALEX far-UV minus near-UV (FUV - NUV) colour-redshift relation is reliably determined up to $z \approx 2.8$, corresponding to rest-frame wavelengths as short as $400 \AA$. Extreme UV (EUV) colours are substantially redder than found previously, when detection limits are properly accounted for. Quasar template spectra were forward modelled through the GALEX bandpasses, accounting for intergalactic opacity, intrinsic reddening, and continuum slope variations. Intergalactic absorption by itself cannot account for the very red EUV colours. The colour-redshift relation is consistent with no intrinsic reddening, at least for SMC-like extinction. The best model fit has a FUV continuum power-law slope $\alpha_{v \text {, FUV }}=$ $-0.34 \pm 0.03$ consistent with previous results, but an EUV slope $\alpha_{v}$, EUV $=-2.90 \pm 0.04$ that is much redder and inconsistent with any previous composite value (all $\gtrsim-2.0$ ). The EUV slope difference can be attributed in part to the tendency of previous studies to preferentially select UV brighter and bluer objects. The weak EUV flux suggests quasar accretion disc models that include outflows such as disc winds.
\end{abstract}

Key words: methods: statistical-catalogues - quasars: general-ultraviolet: general.

\section{INTRODUCTION}

Quasars are the most luminous stable objects in the Universe, and are an important if not dominant source of ionizing radiation throughout much of cosmic history. In particular, the extreme ultraviolet (EUV) to $\mathrm{X}$-ray continuum flux from quasars and other active galactic nuclei (AGNs) is thought to regulate the emission line spectra of the AGNs (e.g. Mathews \& Ferland 1987; Moloney \& Shull 2014), and the evolution of the ionization and thermal state of the intergalactic medium (IGM; e.g. Shull et al. 2004; Haardt \&
Madau 2012; Upton Sanderbeck et al. 2018). The quasar central engine is widely believed to be powered by accretion from a viscous disc on to a supermassive black hole. These models are supported by broad agreement between the observed and predicted optical/UV continuum spectra of quasars (e.g. Shields 1978; Sun \& Malkan 1989). However, in detail the model results are discordant with observed spectra, especially at $\operatorname{EUV}(\lambda \lesssim 1000 \AA)$ to $\mathrm{X}$ ray frequencies. Model spectra are generally too luminous in the EUV, and they turn over at a wider range of frequencies than is observed (e.g. Koratkar \& Blaes 1999). More sophisticated models show great sensitivity in the EUV to the model specifications and parameter values (e.g. Hubeny et al. 2000; Capellupo et al. 2015). 
The EUV regions of quasar spectra therefore provide strong tests of quasar models, and the ionization models that depend upon them. Unfortunately the EUV region is virtually impossible to observe at very low redshift, even from space, due to Galactic absorption in the $13.6 \mathrm{eV}$ to $0.2 \mathrm{keV}$ energy range, and intergalactic absorption dominates the rest-frame EUV at high redshifts. Most of what is known about the EUV region comes from observations of several hundred intermediate-redshift quasars with UV spectra obtained with space-based telescopes such as the Hubble Space Telescope (HST; e.g. Zheng et al. 1997), and the Far-Ultraviolet Spectroscopic Explorer (FUSE; Scott et al. 2004). The spectra of these objects typically exhibit a break near $\approx 1000 \AA$, below which the observed continua become shallower (redder) than in the far-UV (FUV)/optical region. The EUV spectra are generally redder than predicted by standard accretion disc models (e.g. Shang et al. 2005).

The observed EUV continuum is reasonably well described by a power law, with a frequency index given by $F_{v} \propto v^{\alpha_{v} \text { EUv }}$. Composite spectra from a number of studies exhibit indices with values ranging from about -0.6 (bluest value; Scott et al. 2004) to around -2.0 (reddest value; Zheng et al. 1997; Barger \& Cowie 2010). This wide range is in contrast to the optical to FUV region, where composite spectral slopes differ by at most a few tenths (e.g. Vanden Berk et al. 2001; Selsing et al. 2016). Various explanations have been proposed to account for the wide EUV variation, including different continuum measurement windows, different methods of correction for absorption by the IGM, possible dependence upon luminosity, and small sample sizes. Sample selection variations likely also contribute to the slope dispersion, since the majority of quasars with UV spectra were observed for purposes unrelated to describing the quasar UV continuum (e.g. to provide bright UV background probes for absorption line studies). Larger samples with known selection functions are needed to confidently characterize the EUV emission from quasars.

The Galaxy Evolution Explorer (GALEX; Martin et al. 2005) satellite has allowed a complementary approach to the study of the EUV region of AGNs (Trammell et al. 2007). Instead of obtaining UV spectroscopy of a relatively small number of objects, a very large number of objects can be observed photometrically in two UV bands. Centred at roughly $1500 \AA$ (FUV) and $2300 \AA$ (NUV), observations in these bands correspond to quasar rest-frame EUV wavelengths at redshifts $z \gtrsim 0.3$. Rest wavelengths below $\approx 700 \AA$ are covered by UV spectra of only about 100 quasars, but this region is potentially explored with reliable GALEX photometry of tens of thousands of known quasars.

The GALEX photometry of large samples of quasars have been incorporated in the construction of spectral energy distributions (SEDs) that cover some part of the EUV (Trammell et al. 2007; Krawczyk et al. 2013; Scott \& Stewart 2014). The far to extreme UV region in those SEDs is generally consistent with a peak near $\sim 1000 \AA$, roughly in agreement with spectroscopic results. However, the SEDs do not extend much below about $800 \AA$, and absorption by the IGM was accounted for in only the Krawczyk et al. (2013) study. Another potentially serious limitation of these studies is that only quasars with GALEX detections were included in the UV SEDs. Detected objects will generally be brighter, and therefore bluer in the UV for a given optical detection limit, which would bias the quasar samples in favour of harder EUV spectral slopes. Careful consideration of sample selection, absorption correction, and detection limits are needed before GALEX photometry can reliably constrain the quasar EUV region.

The largest single source of verified quasars is the Sloan Digital Sky Survey (SDSS; York et al. 2000), which has reported over a half-million quasars in the Data Release 14 (DR14; Abolfathi et al. 2018) quasar catalogue (DR14Q) described by Pâris et al. (2018). The GALEX UV survey covered the vast majority of the SDSS sky area, resulting in UV observations of over 480000 SDSS quasars. In this study, we cross-match the DR14Q sample with the final GALEX GR6/7 photometric catalogue, and we include estimates of upper UV detection limits (lower magnitude limits) for every covered quasar. The detection limits are accounted for in the analysis of the median UV colour trends with redshift. Quasar spectra that are consistent with the corrected colour trends are found by forward modelling template spectra through the GALEX bandpasses, including opacity of the IGM, and possible attenuation by dust intrinsic to the quasars.

The paper is organized as follows. The SDSS quasar sample and matching to the GALEX photometry are described in Section 2. That section includes analysis of the catalogue astrometric matching (Section 2.2.2), GALEX source exclusion (Section 2.2.3), GALEX detection probabilities (Section 2.2.4), GALEX detection limits (Section 2.2.5), and UV contamination by nearby SDSS sources (Section 2.2.6). Median colour-redshift relations, accounting for detection limits, are calculated and fit with model template quasar spectra in Section 3. A comparison is made with previous EUV spectral studies in Section 4. The results are discussed in relation to the expectations from theoretical quasar models in Section 5. The conclusions are summarized in Section 6.

\section{QUASAR SAMPLE AND SDSS MATCHES TO GALEX PHOTOMETRIC DATA}

\subsection{Sloan Digital Sky Survey DR14Q quasar sample}

All of the quasars are drawn from the Sloan Digital Sky Survey (SDSS; York et al. 2000) Data Release 14 (DR14; Abolfathi et al. 2018) quasar catalogue (DR14Q) described by Pâris et al. (2018). That catalogue contains descriptive information for 526356 spectroscopically confirmed quasars discovered at any stage of the SDSS through DR14. The quasars were selected as candidates for spectroscopic verification based upon a variety of criteria, which are described by Pâris et al. (2018) and references therein. Of particular interest for this study is the substantial increase in $z \lesssim 2$ quasars, compared to other recent data releases (e.g. DR12Q; Pâris et al. 2017), since the fraction of quasar detections by GALEX is a strong function of redshift (Trammell et al. 2007). Sub-samples of quasars can be readily constructed based upon a large number of available parameters and target selection flags.

\subsection{GALEX matching data}

\subsubsection{GALEX mission}

GALEX (Martin et al. 2005) is a NASA satellite mission to provide UV images of most of the sky in two bands, labelled FUV and NUV, centred roughly at 1540 and $2320 \AA$, respectively. The calibration and general performance of GALEX are described by Morrissey et al. (2007). During the course of its mission, GALEX covered most of the sky area surveyed by the SDSS. The overlap with the SDSS area is not complete, because the satellite was not able to point to all sky locations, due to the presence of bright stars and other limitations. GALEX was decommissioned in June 2013, after more than a decade of operation. UV data for each quasar, when available, was retrieved from the final GALEX data release, GR6/7, 
which is publicly available through the Mikulski Archive at the Space Telescope Science Institute (MAST) website. ${ }^{1}$

The SDSS DR14Q catalogue provides FUV and NUV flux estimates and variances, based upon force-photomering of the GALEX image data at the astrometric locations of the SDSS quasars. Although this photometry method provides a flux value for every quasar covered by GALEX, we have decided to use instead the photometric information provided in the GR6/7 catalogue. At present, there is too little information accompanying the forcephotometered fluxes, such as the tile information, exposure times, and processing flags, for the purposes of the current study. However, the force-photometered fluxes may offer several advantages once they are more fully characterized. For those interested in the comparison, the following equations provide matches good to faint magnitudes, between the GALEX catalogue magnitudes and the force-photometered fluxes, nuvFlux and fuvFlux

$\mathrm{NUV}=-(2.5 \log e) \operatorname{asinh}($ nuv Flux $/ 0.01)+28.3$,

$\mathrm{FUV}=-(2.5 \log e) \operatorname{asinh}(f u v F l u x / 0.01)+28.4$.

The asinh magnitude system (Lupton, Gunn \& and Szalay 1999) is used here because many of the reported fluxes fall below zero.

The GALEX sky coverage and imaging depth is not uniform across the sky. GALEX imaging data has been classified into several 'surveys,' including the All-sky and Medium Imaging Surveys (AIS and MIS, respectively), depending largely on the tile exposure time, and the purpose of the observations. Tile areas are circular in shape, and necessarily overlap to cover contiguous regions of the sky. The overlap pattern and exposure time distribution is complex. Budavári et al. (2009) addressed these issues with a geometric approach that resolved the AIS and MIS into nonoverlapping contiguous sky regions. (See Bianchi, Shiao \& Thilker 2017, for a related approach focusing on the AIS data.) Multiple detections of objects in the overlapping regions were then classified as primaries, if they were counted in the resolved region, or secondaries otherwise. This allows the construction of catalogues with detected objects listed only once. It also avoids biases that might be introduced if object selection were based on signal-to-noise ratio $(\mathrm{S} / \mathrm{N})$.

In this study, we use a different approach based upon effective exposure time (rather than geometry or $\mathrm{S} / \mathrm{N}$ ) that can be readily applied to the existing searchable GALEX data base. For each SDSS object of interest, the set of GALEX tiles that cover its coordinates is retrieved from the GALEX data base. Data for the nearest neighbour to the coordinates on each tile considered independently is extracted from the data base. The data that is kept for each object corresponds to that data from the tile in which the object would have the longest effective exposure time, regardless of whether the object was detected, and independent of the $\mathrm{S} / \mathrm{N}$ of any detection. This is equivalent to selecting the longest blind observation of each object, which is independent of the properties of the object. The details of this process will be described in the following sections.

Whether or not a quasar was detected by GALEX depends upon both observational details and the physical properties of the quasar. The GALEX effective exposure time, angular proximity of a quasar to other sources, quasar brightness, and redshift are examples of factors that contribute to the detection probability. Each of these factors is considered in the following sections.

\footnotetext{
${ }^{1}$ galex.stsci.edu
}

\subsubsection{Astrometric matching}

The absolute astrometric precision of the SDSS is better than 0.1 arcsec (Pier et al. 2003), which is much smaller than the expected value for GALEX. GALEX astrometry therefore has been tied to the SDSS system. For the GR2/GR3 data release, the GALEX absolute astrometry was reported to be 0.59 arcsec for NUV detections of SDSS quasars with S/N larger than 10 (Morrissey et al. 2007). We have made a similar measurement of the astrometric accuracy and precision of the GR6/7 data release, using the much larger available sample of SDSS quasars.

The relative GALEX to SDSS astrometry consistency was checked with a sub-sample of quasars that are highly likely to be detected with GALEX, at least in the NUV band. Quasars with SDSS $u$-band magnitudes brighter than 21.0, and redshifts $z \leq 1.5$ were selected for the comparison. The vast majority of such quasars are expected to be detectable in GALEX images, even at the effective exposure times of the AIS $\left(t_{\mathrm{eff}} \sim 100 \mathrm{~s}\right)$ (Trammell et al. 2007). This results in a sub-sample of over 107000 objects.

The GALEX casjobs SQL server was used to identify all tiles covering each of the quasars in the sub-sample. Only tiles covering a coordinate less than $0.59 \mathrm{deg}$ away from the tile centre were selected, since the image quality beyond that distance on a tile degrades rapidly. On average, a typical quasar was covered by just over two tiles.

Next, information was extracted for the GALEX object nearest in angular separation to each quasar coordinate on each covering tile. No restriction was made to the separation of the quasar and GALEX detection coordinates, since one of the goals here is to determine how the detection fraction depends on separation. In every case of multiple covering tiles, the nearest neighbour with the longest effective exposure time was selected, again, regardless of separation. The vast majority of the GALEX detections should correspond to the quasars in the sub-sample, so statistical descriptions of the astrometric matches are assumed to be reliable.

The distribution of angular offsets of GALEX nearest neighbour positions, relative to the corresponding SDSS quasar positions, is shown in Fig. 1. The offsets are shown in relative equatorial coordinates. A small but significant systematic offset of the GALEX positions is evident to the north-east, of approximately 0.32 arcsec (median values of 0.16 arcsec eastward and 0.28 arcsec northward). The rms dispersion is nearly the same in both directions at 0.78 arcsec. The values do not change appreciably when considering only objects classified as extended or point-like in the SDSS images. Both the magnitude of the offset and the dispersion of separations are similar to those reported by Morrissey et al. (2007).

For many purposes the small (but significant) median offset is not worth attempting a correction. However, since part of the goal here is to reliably cross-match a large number of objects, even a small offset may cause many true matches to be excluded, and include many spurious matches. One approach to account for the offset is to assume that the SDSS and GALEX coordinate systems differ simply by a set of small rotations. In fact, we have found that to avoid increasing the offset dispersion, at least two separate sets of rotations are needed - one is applied to objects in the northern Galactic cap (NGC) and one in the southern Galactic cap (SGC). Because the object catalogue matches are carried out from SDSS to GALEX coordinates, we have determined the transformations from the SDSS to GALEX system, to simplify the cross-matching. We assume the rotation angles are small, and 


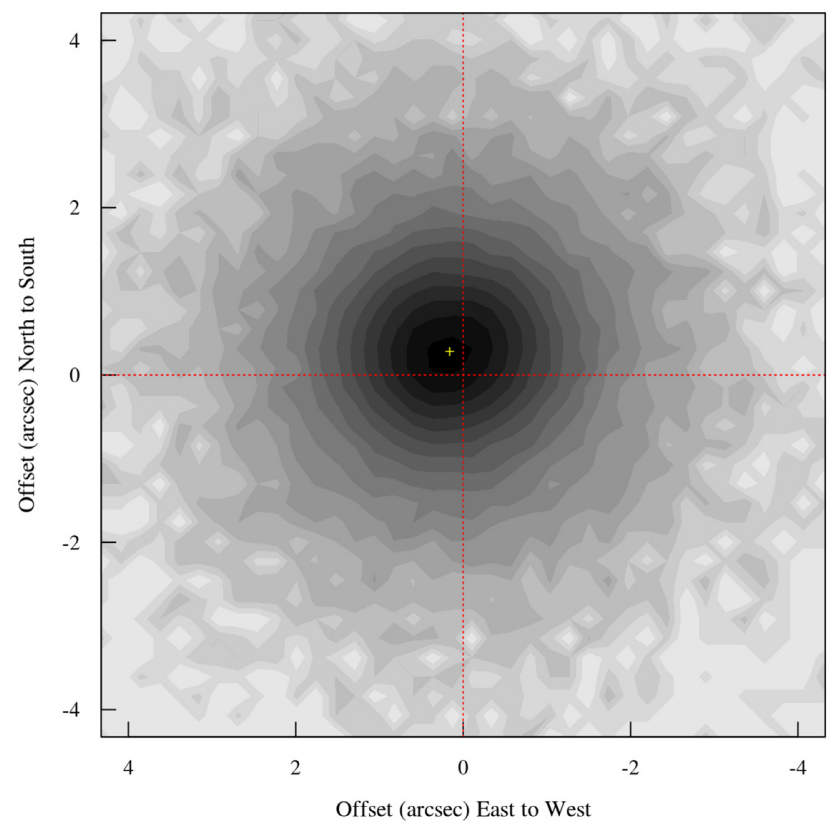

Figure 1. Angular distribution of GALEX nearest neighbour coordinates relative to input SDSS quasar coordinates. The contours are logarithmically spaced. The median value of the distribution, indicated by the yellow ' + ', is offset from the origin by 0.162 arcsec eastward, and 0.276 arcsec northward (with positional uncertainty of 0.003 arcsec in each direction), consistent with the GALEX to SDSS offset reported by Morrissey et al. (2007).

that a transformation can be described with the following matrix equation,

$\mathbf{P}_{\text {GALEX }}=\left(\begin{array}{ccc}1 & -\psi & \theta \\ \psi & 1 & -\phi \\ -\theta & \phi & 1\end{array}\right) \mathbf{P}_{\mathrm{SDSS}}$,

where $\mathbf{P}_{G A L E X}$ and $\mathbf{P}_{\text {SDSS }}$ are vectors describing the equatorial coordinates of a point on the unit sphere in the GALEX and SDSS systems, respectively, and $\psi, \theta$, and $\phi$ are the rotation angles. The values of the rotation angles for the NGC are $(\psi, \theta, \phi)=(0.34,0.26$, $0.05 \operatorname{arcsec})$, and for the SGC are $(-0.22,-0.38,-0.07 \operatorname{arcsec})$, found by minimizing the median offset in both the East-West and North-South equatorial directions. Uncertainties in the angles are about 0.01 arcsec, determined by recalculating the angles for numerous random subsets of quasar coordinates. These transformations (using angle units of radians in the calculations) bring the median offset positions to almost zero, without significantly changing the dispersion. In the following analysis, all of the SDSS coordinates were transformed to the GALEX system using equation (3).

The dispersion of the position offsets in the two systems depends on the quality of the GALEX images of the objects. For example, Figs 2 and 3 show the standard deviations of the astrometric offset distributions as functions of distance from a tile centre, and NUV magnitude. In each case the standard deviations remain at roughly 0.7 arcsec for most of the parameter ranges, then rise to nearly twice that value for the objects that are the faintest, or are closest to the edge of an imaging tile. The dispersion trends in the East-West and North-South directions are similar in all cases. The dependence of the distribution of offsets on various quantities, shows that a single matching search radius may not be adequate to determine if an SDSS object is truly detected in GALEX imaging.

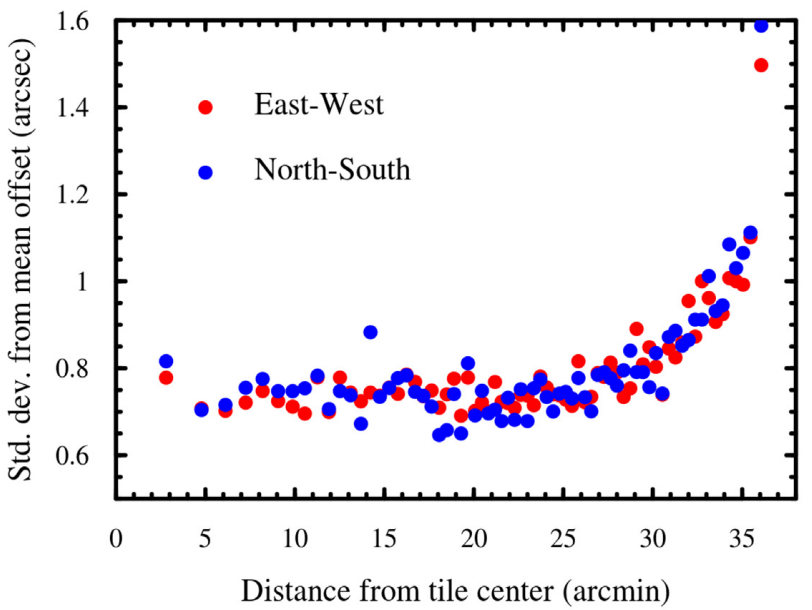

Figure 2. Standard deviation of the GALEX versus SDSS astrometric offset distribution in the East-West (red) and North-South (blue) directions, as a function of distance from a GALEX tile centre. The standard deviation is measured relative to the mean offset positions in each direction. Each point represents the results for a sample of 1000 quasars.

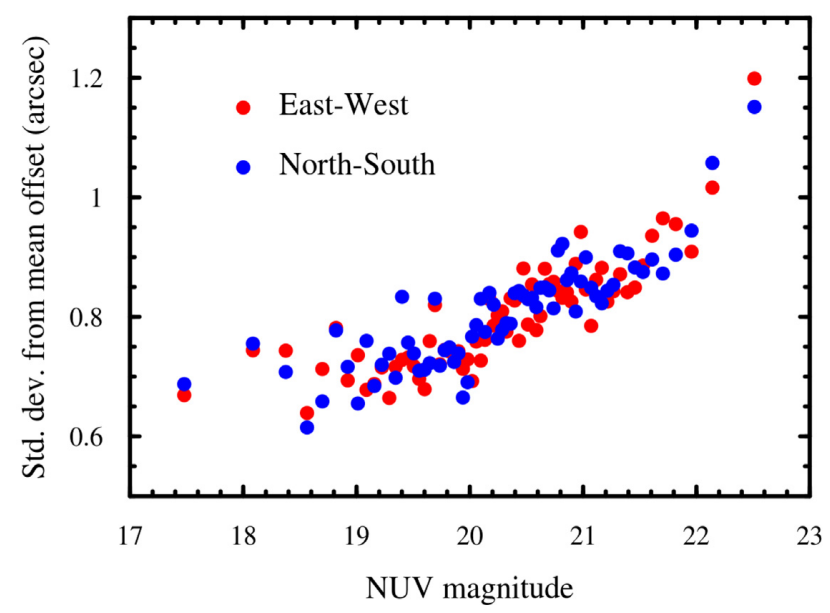

Figure 3. Standard deviation of the GALEX versus SDSS astrometric offset distribution in the East-West (red) and North-South (blue) directions, as a function of GALEX NUV magnitude. The standard deviation is measured relative to the mean offset positions in each direction. Each point represents the results for a sample of 1000 quasars.

The detection probability as a function of search radius is examined further in Section 2.2.4.

\subsubsection{GALEX source exclusion}

The GALEX point spread function (PSF) is reported to have a width of 5.3 arcsec in the NUV band and 4.2 arcsec in the FUV band (Morrissey et al. 2007). Those values are several times larger than the SDSS PSF widths, so there is the possibility that multiple SDSS sources can be blended into what appears to be a single GALEX detected object, and there will be some region around a detected object in which no other objects will be detected. The source exclusion profile - the probability that another object will be detected as a function of the separation from a detected object - can be constructed from a sample of detections and their neighbours. This is related to, but not identical with the problem of source 


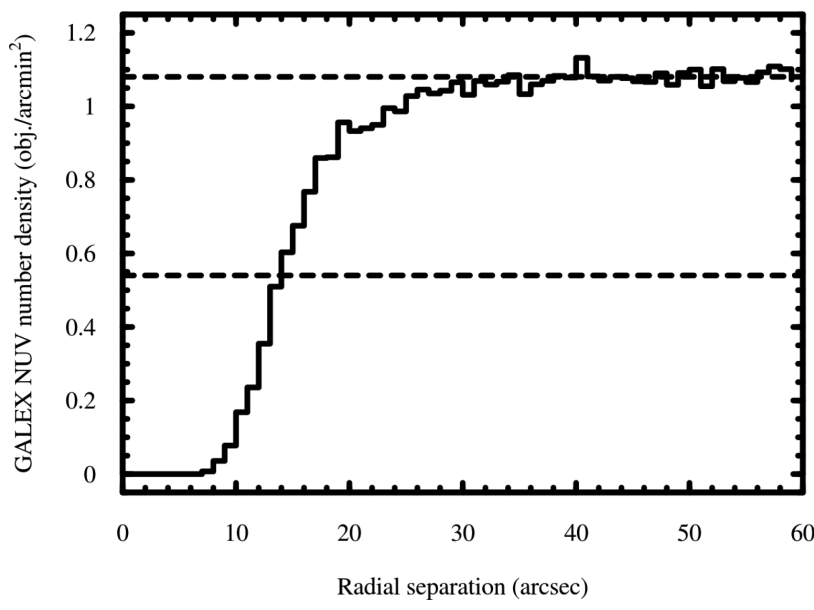

Figure 4. Solid angular number density of neighbours to GALEX coordinates of reliably detected SDSS quasars as a function of radial separation. The density is shown in separation bins of 1 arcsec. The density at large separations ( $\gtrsim 40$ arcsec) is nearly constant at about $1.08 \mathrm{arcmin}^{-2}$, and is shown with the upper dashed line. The density of detected objects reaches half of the large-separation density (indicated by the lower dashed line) at about 15 arcsec. The large-separation density is not reached until a separation of over 30 arcsec. Objects nearer to each other than that may be recorded as a single object in the GALEX catalogue.

confusion in crowded GALEX fields (e.g. Hammer et al. 2010; Worseck \& Prochaska 2011). The sample selection for this analysis consists of highly likely GALEX NUV detections of SDSS quasars, with coordinate separations less than 3 arcsec, within 32 arcmin of a tile centre, and with effective exposure times between 50 and $250 \mathrm{~s}$. The effective exposure time range brackets the AIS-like tiles, from which the majority of quasar detections is drawn. For objects covered by multiple tiles, the detection with the smallest distance to its tile centre was selected. About 87000 objects are in the sample. All of the neighbours to the objects on the same tiles were found in the GALEX data base, out to a search radius of 1 arcmin. The solid angular number density of neighbours as a function of radial separation out to 60 arcsec from a quasar NUV detection is shown in Fig. 4.

The solid angular density of the neighbours is statistically flat (at about 1.08 objects per $\operatorname{arcmin}^{2}$ ) at separations beyond about $35 \operatorname{arcsec}$ (Fig. 4). At smaller separations the density is lower, and the probability of detecting a neighbour of a detected object (the ratio of the true density to the large-separation density) can be very low. In fact, the probability of detecting a neighbour is consistent with zero out to separations of $7 \mathrm{arcsec}$. The probability is 50 per cent at about 15 arcsec, and it does not reach unity until separations just over 30 arcsec. This detection exclusion profile does not change significantly with effective exposure time (although the average large-separation density increases with exposure time). However, the width of the profile increases with NUV brightness: for NUV magnitudes between 21 and 22 the 50 per cent detection probability occurs at about 12 arcsec; the separation for the same probability rises steadily to 17 arcsec for objects with NUV magnitudes between 18.0 and 18.5 .

The rather wide exclusion profile can pose problems for studies that attempt to match known objects to GALEX detections. Among any group of detectable objects closer to each other than about 15 arcsec (depending on NUV magnitude) only one object is likely to be detected. A rough estimate follows of the fraction of detectable objects that are not detected, due to the exclusion regions. The probability that at least one neighbouring object is within an angular distance $\theta$, of a quasar is $1-\mathrm{e}^{-\pi \theta^{2} n}$, where $n$ is the average solid angular number density of detectable (not detected) background objects. If a neighbour within the exclusion radius results in a quasar being undetected half the time, then the probability that a quasar is undetected is

$1-\frac{n_{\mathrm{det}}}{n}=0.5\left(1-\mathrm{e}^{-\pi \theta^{2} n}\right)$,

where $n_{\text {det }}$ is the number density of detected objects. For an effective exclusion radius of $\theta=15 \mathrm{arcsec}$, and a detected object number density of $n_{\text {det }}=1.08 \mathrm{arcmin}^{-2}$, the detectable number density is $n=1.21 \operatorname{arcmin}^{-2}$, and the probability that a quasar will not be detected due to a nearby neighbour is 0.11 . So on AIS-like tiles, approximately 10 percent of detectable quasars will be missed because they lie too close to another detected object. Denser sky regions, or tiles with longer exposure times, will generally yield more detectable objects, so the fraction of non-detected quasars will be larger there.

We have inspected the GALEX images of a number of close quasar pairs to better understand the source exclusion issue. For most examples, the quasars are clearly visibly distinguishable as separate objects, and not so blended as to appear as 1. A typical example is the quasar pair SDSS J001257.25+011527.3 and SDSS J001257.89+011528.0 with a separation of $9.5 \operatorname{arcsec}$, and $u$ magnitudes of 19.0 and 19.7, respectively. The objects are covered by five GALEX tiles. On each tile both objects are clearly seen as two distinct objects. The first object is reported as detected in the GALEX catalogue on all five tiles, in both the FUV and NUV bands. However, the second object is reported as detected on only two of the tiles, and only in the NUV band.

Clearly then, caution should be exercised when using the GALEX catalogue results for the analysis of objects with small angular separations. Close quasar pairs, which are important e.g. for clustering and gravitational lensing studies, will be heavily affected, even out to separations as large as five times the width of the GALEX NUV PSF. For these studies, other image detection and extraction techniques, such as forced photometry, should be considered. While quasars were used as reference objects in this analysis, the results should hold for stars or galaxies in the same sky regions. The effect of neighbours on the photometric properties of quasars in GALEX images is explored in Section 2.2.6.

\subsubsection{Detection probability}

A GALEX detection in close proximity to an SDSS quasar coordinate may be a true detection, or a spurious match to an unrelated object. In this section the probability that a GALEX neighbour to an SDSS quasar coordinate is a true UV detection of the quasar is determined, as a function of coordinate separation. The probability was assessed from the sample of all GALEX nearest neighbour matches to quasar coordinates, compared to a nearest neighbour sample to randomly offset coordinates. Because the exclusion profile around detected GALEX objects far exceeds the likely matching radius around SDSS coordinates (cf. Section 2.2.3 and Fig. 1), there will be no cases of multiple GALEX candidates for quasar matches on individual tiles.

The sample of all GALEX nearest neighbours was generated by finding the nearest neighbour to every quasar coordinate on every overlapping GALEX tile, out to a limiting radius of 30 arcmin. Just under 92 percent of the SDSS quasars were covered by at 


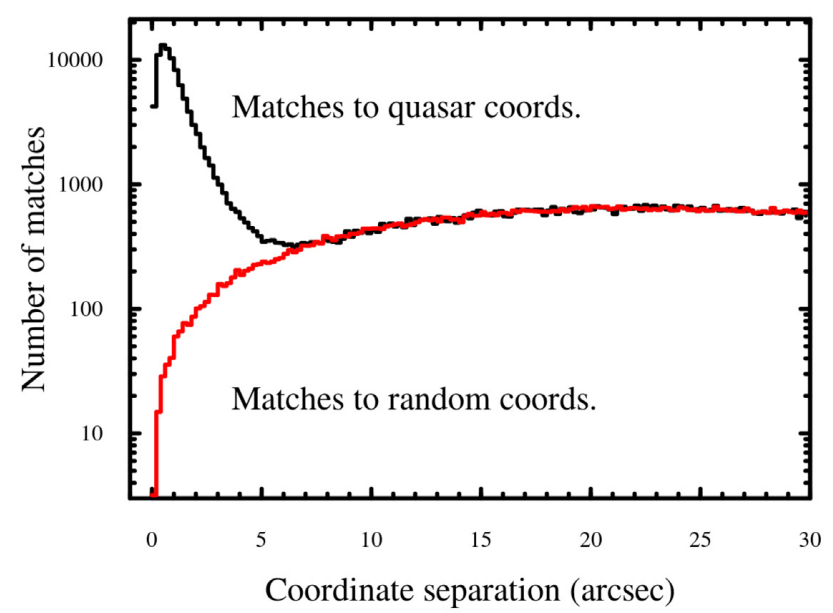

Figure 5. Distribution of separations of nearest neighbour GALEX matched objects to SDSS quasar coordinates, for AIS-like tiles (black). The distribution of separations of GALEX nearest neighbours to coordinates randomly offset from SDSS positions is shown in red, scaled to match the true distribution at large separations. The width of each separation bin is 0.2 arcsec.

least one GALEX tile. For quasars with neighbours on multiple overlapping tiles, the neighbour on the tile with the largest effective exposure time (in each band separately) was selected for the sample. Thus, the sample contains at most one GALEX neighbour for each quasar coordinate. The distribution of radial separations between the GALEX neighbour and SDSS quasar coordinate is shown in Fig. 5, for AIS-like tiles. The distribution peaks sharply at about 0.6 arcsec, then drops to a minimum at about 7 arcsec, beyond which the distribution slowly rises to a plateau. True matches dominate the distribution at smaller separations, while spurious matches eventually take over at larger separations.

The probability that a nearest neighbour is a true quasar detection can be determined by comparing the real separation distribution to the distribution for nearest neighbours to random coordinates. The quasar coordinates were offset by 1 arcmin in random directions, and the GALEX nearest neighbours were found. The distribution of separations to the random coordinates was scaled to match the true nearest neighbour distribution at large separations. The resulting scaled distribution, shown in Fig. 5, represents the number of nearest neighbours to real coordinates that are expected to be spurious matches.

The difference between the number of neighbours to the real coordinates and to the random coordinates, at each separation, gives the distribution of separations to the true quasar matches. The ratio of this difference to the number of all (real coordinate) nearest neighbours gives the probability that a nearest neighbour is a true quasar match, as a function of radial coordinate separation. This probability, for the AIS-like tiles in the NUV band, is shown in Fig. 6. The probability of a true detection is near 100 per cent up to separations of about 1 arcsec. The probability drops to 50 per cent at 4.6 arcsec, and is consistent with zero at separations of about 7 arcsec. Thus, there is a significant probability that a nearest neighbour detection is a true quasar match out to separations well beyond 3 arcsec, which is a common value used as an upper limit to search for GALEX matches to SDSS objects. The radial probability profile for the FUV band is similar.

The cumulative fraction of all true matches to SDSS quasars as a function of radial separation is also shown in Fig. 6. Just

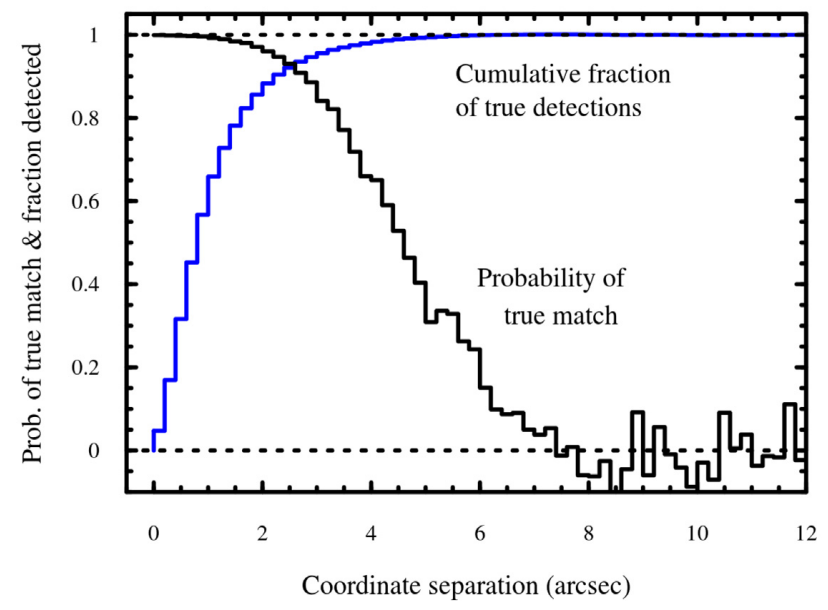

Figure 6. The cumulative fraction of true GALEX quasar detections (blue), and the probability that a GALEX detection is truly that of a quasar (black), as functions of separation from SDSS quasar coordinates.

over 95 percent of detected quasars are found at separations less than 3 arcsec. For the full DR14Q sample, this means that about 12000 quasars detected by GALEX will be missed in cross-matching searches limited to separations of 3 arcsec. The statistically determined total number of observed quasars that have true matches, is the difference between the number of all neighbours and the estimated number of spurious neighbours (e.g. the area between the two curves in Fig. 5). For the full SDSS quasar sample, 49.3 per cent (about 238000 ) of the quasars are detected in the NUV band, and 22.0 per cent (about 104000) are detected in the FUV band. The detection probability depends strongly upon other quantities such as redshift, optical magnitude, and optical colour; these dependencies are discussed in Section 2.3.

\subsubsection{Upper GALEX magnitude limits}

For statistical studies of the UV properties of quasars, it is often necessary to know the limiting magnitudes of the quasars that have been observed but not detected. Typically, the limiting magnitude of an imaging survey is defined as the magnitude at which the number of detected objects per unit magnitude is half of the number expected to have been observed. However, we do not know the expected number density of quasars in the GALEX UV bands. Instead, here we take advantage of the fact that a large fraction (nearly $2 / 3$ ) of the quasars have been observed multiple times with GALEX, at different exposure times. The sample of quasars detected in the longest observation of each quasar, serves as the comparison sample for the shorter observations.

Effective exposure time values $t_{\text {eff }}$, are available in the GALEX catalogue only for detected objects. For this study, the $t_{\text {eff }}$ value of the closest neighbour to the SDSS coordinates was assigned to each quasar, if the neighbour was within $30 \operatorname{arcsec}$ of the coordinate. For the remaining quasars, the average ratio of $t_{\text {eff }}$ to actual exposure time, as a function of distance to a tile centre, was used to scale the actual exposure time of the quasar to a $t_{\text {eff }}$ value.

The comparison sample of quasars in the longest $t_{\text {eff }}$ observations, was constructed by first removing all observations with ambiguous quasar detections. That is, all observations in which the nearest neighbour to the SDSS coordinate is between 3 and 7 arcsec 


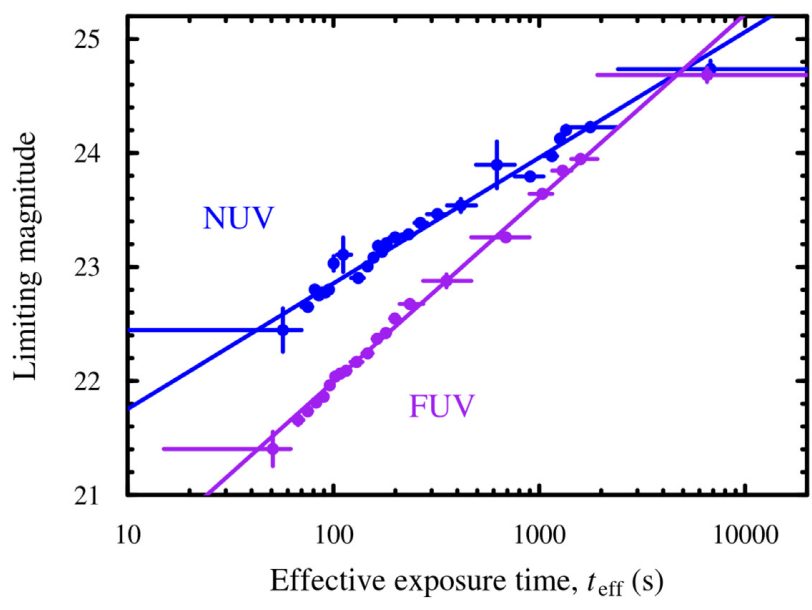

Figure 7. Magnitude limits as a function of effective exposure time for the NUV (blue) and FUV (purple) bands. Line fits to the limiting magnitudes versus $\log \left(t_{\mathrm{eff}}\right)$ are shown for each. Uncertainties on the magnitude limits are found using bootstrap resampling of the multiply-observed quasar sample.

were removed. In this way, the remaining observations result in either a confident quasar detection (separation less than 3 arcsec), or confident non-detection (separation greater than 7 arcsec) (see Section 2.2.4). Also, only quasars with redshifts less than 3 were selected, since the probability of detection at larger redshifts is very small. The comparison sample consists of all quasars with multiple remaining observations, and a confident detection in the longest remaining observation of each quasar. There are 155436 such quasars with NUV observations, and 62663 with FUV observations. There are 271783 and 105041 additional observations of those quasars in the NUV and FUV bands, respectively.

The additional observations were divided into bins of effective exposure time ranges, containing $10^{4}$ observations each. For each exposure time bin, the distribution of magnitudes of the quasars detected in the additional observations, was compared to the distribution of magnitudes in the primary observations (from the comparison sample). The distributions were determined using a Gaussian kernel density estimator, with a kernel width of 0.25 magnitudes. This width was selected because it is the mean value of both the FUV and NUV magnitude errors of the full sample. The magnitude at which the density of quasars in the additional observations was half of the density of the comparison sample, was taken as the limiting magnitude for a given exposure time bin.

The magnitude limits as a function of effective exposure time for both the NUV and FUV bands are shown in Fig. 7. The uncertainties in the magnitude limits were found using bootstrap resampling of the multiply-observed quasar sample. The magnitude limits are well described by line fits to the limits versus $\log \left(t_{\text {eff }}\right)$, as seen in the figure. The line fits to the NUV and FUV magnitude limits are

$$
\begin{aligned}
& \mathrm{NUV}_{\text {lim }}=(1.10 \pm 0.03) t_{\mathrm{eff}, \mathrm{NUV}}+(20.65 \pm 0.07), \\
& \mathrm{FUV}_{\text {lim }}=(1.61 \pm 0.03) t_{\mathrm{eff}, \mathrm{FUV}}+(18.77 \pm 0.06)
\end{aligned}
$$

The precision of the coefficients comes solely from the line fitting; the uncertainty of the limiting magnitude of any particular object will depend also on the uncertainty of its effective exposure time. Magnitude limits were assigned to each quasar observation for which there was no GALEX detection.

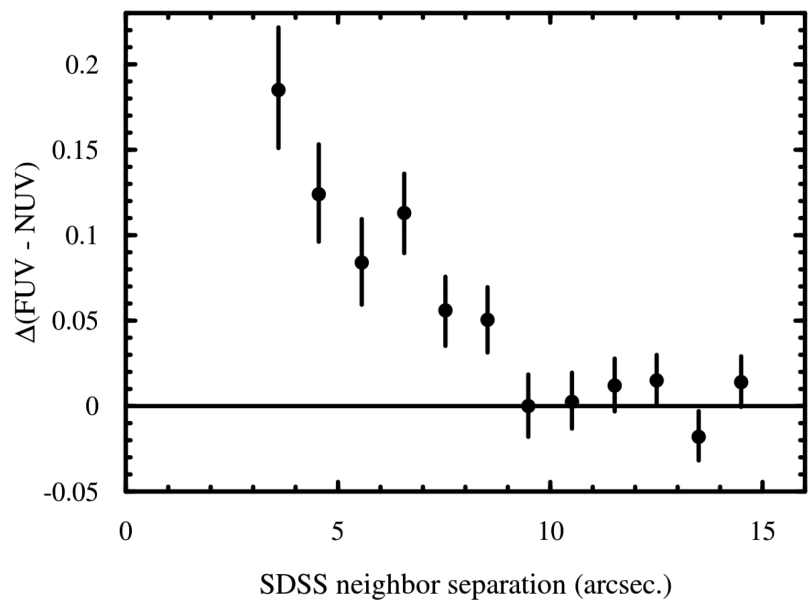

Figure 8. UV colour differences between quasars with and without nearby SDSS neighbours, as a function of neighbour separation. Each quasar with a neighbour was matched to a set of quasars without neighbours, having nearly the same redshifts, $u$ magnitudes, and Milky Way $E(B-V)$ reddening values. The colour differences can be explained by artificially bright NUV magnitudes, caused by neighbour contamination.

\subsubsection{Nearby SDSS neighbours and photometric contamination}

Because of the relatively large PSF of the GALEX images, multiple SDSS objects can often be matched to the same GALEX object (e.g. Budavári et al. 2009). In addition, even when the correct object is matched, the nearby SDSS neighbours can affect the GALEX UV photometry of the object of interest. Budavári et al. (2009) found that approximately 15 per cent of GALEX AIS sources have multiple SDSS neighbours within a 4 arcsec search radius. To assess the effect of nearby neighbours on the UV photometry of quasars, reliably detected neighbours to all of the quasars in the SDSS DR14 photoprimary table were found, to a radius of 15 arcsec (the mid-point of the source exclusion profile, Section 2.2.3). The number of quasars with at least one SDSS neighbour rises steadily to over 70 per cent at a separation of 15 arcsec, including both pointlike and extended objects, which contribute roughly equally.

The FUV - NUV colour differences between quasars with and without nearby SDSS neighbours is shown in Fig. 8, as a function of neighbour separation. The colour difference is defined as the median difference between the colours of quasars with a single neighbour, and the colours of isolated quasars that were matched to have nearly the same redshifts, $u$ magnitudes, and Galactic $E(B-$ $V)$ values. Just over 10000 quasars with a single neighbour were able to be matched with at least one isolated quasar. Colour differences were calculated in separation bins of 1 arcsec; too few quasars have SDSS neighbours within 3 arcsec for reliable calculations there.

Fig. 8 shows that nearby neighbours affect the FUV - NUV quasar colours, making them appear redder than they would be without nearby neighbours; that is $\Delta(\mathrm{FUV}-\mathrm{NUV})>0$ for neighbour separations up to about 10 arcsec. A simple explanation for this is that light from nearby neighbours causes the NUV magnitudes to appear brighter, while having little effect on FUV magnitudes. Most objects detected in SDSS imaging have weak, if any detectable FUV fluxes, so relatively little contamination might be expected in FUV photometry. A similar analysis of NUV $-u$ colour differences shows that quasars with neighbours are slightly bluer in that colour than those without neighbours, which would be expected if the NUV magnitudes are artificially brightened by neighbour contamination. 
The extent to which neighbour contamination affects a study will depend upon the details of the study. Not all neighbours will contaminate the UV photometry, and the likely benign cases may be identified by cuts in SDSS colour-magnitude space. Of course, for individual objects of interest, it will still be prudent to carefully inspect the images and their surrounding regions. In the SDSSGALEX quasar catalogue, we report the counts of SDSS neighbours to each quasar, to a separation of 15 arcsec. Also included are the separation and $u$ magnitude of the nearest SDSS neighbour if it lies within 15 arcsec.

\subsection{Ultraviolet detection completeness}

Of the initial set of 526356 quasars in the DR14Q, 91.8 percent (483 161) were observed at least once by GALEX. Slightly more quasars were observed in the NUV band than the FUV band (91.8 percent and 89.4 percent, respectively). There are 264812 NUV matches (54.8 percent) and 111594 FUV matches (23.7 percent) within 7 arcsec of the rotated SDSS coordinates. Because the detection probability depends upon match separation (Section 2.2.4), not all of the matches are true UV detections of the SDSS quasars. Aside from the separation and effective exposure time, the detection rates will depend on the quasar optical magnitude and redshift (Trammell et al. 2007), since the UV magnitudes are correlated with those quantities.

To determine the detection rate as a function of various quantities, we avoid cases in which a detection is ambiguous, by ignoring all quasars with UV neighbours within 3 and 7 arcsec. Neighbours closer than 3 arcsec are highly likely to be true detections, while no detections are expected beyond 7 arcsec (Section 2.2.4). Effectively, the statistics are calculated as if quasars with UV neighbours inside this exclusion ring were not observed. We have also excluded quasars with SDSS neighbours within 10 arcsec, to avoid any spurious detections or photometric contamination. The results below do not change appreciably when quasars with SDSS neighbours are included. Quasars with identified broad absorption lines (BALs) are also excluded. Detection rates are found by dividing the number of observed quasars by the number of detected quasars, in small bins of the quantity of interest.

Fig. 9 shows the detection rate as a function of SDSS $u$ magnitude, for all effective exposure times. The NUV and FUV detection rates are nearly 100 per cent at bright magnitudes. With increasing magnitude, the rates drop down to very low values, and then flatten at $u$ magnitudes of about 24 and 22 for the NUV and FUV bands, respectively. The long tails at faint $u$ magnitudes are not due to spurious matches, since we have excluded quasars with SDSS neighbours. Instead, the tails are probably the result of uncertain $u$ magnitude values. The SDSS $u$ magnitudes are highly unreliable beyond $u \approx 22.5$, which effectively becomes a lower magnitude limit. This is because of the nature of the 'asinh' magnitude system used in the SDSS, which gives real-valued magnitudes, even for zero or negative measured flux values (Lupton et al. 1999). The $u$ magnitude distribution is therefore much wider than the true $u$-band flux distribution, which makes it appear as though the UV detection rate has a long faint tail.

The detection fraction as a function of redshift is shown in Fig. 10. In this case, the $u$ magnitude was restricted to be less than 21.0, which is bright enough that the vast majority of low-redshift $(z \lesssim 0.7)$ quasars should be detected in the NUV and FUV bands, at least in MIS-like images (Trammell et al. 2007). Here, AIS-like and MIS-like observations are defined by effective exposure times ranging from 60 to 400 and 700 to $3600 \mathrm{~s}$, respectively. The MIS-like

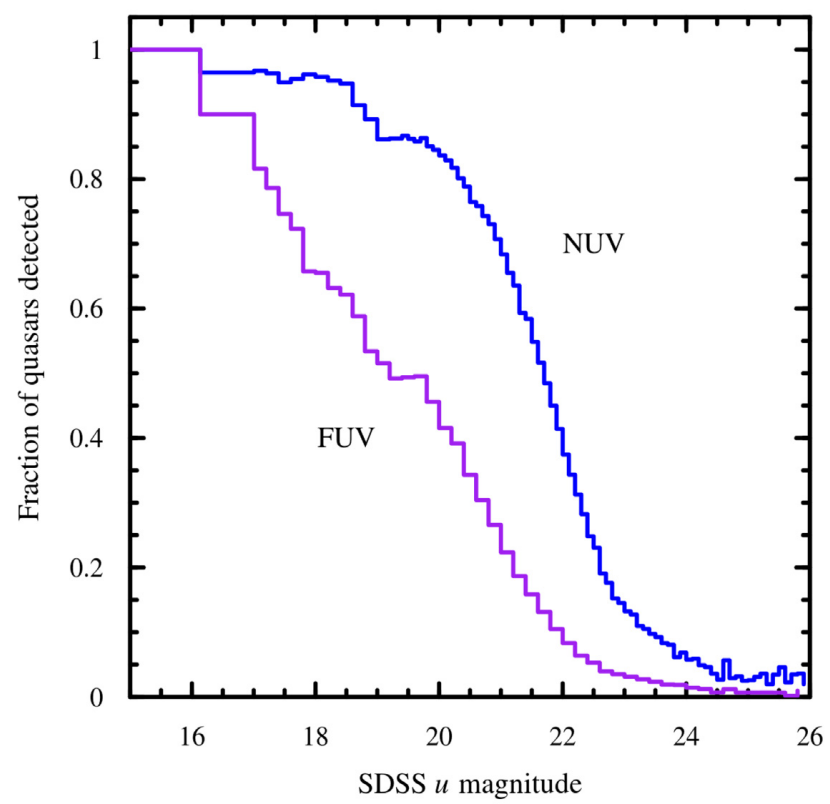

Figure 9. The GALEX quasar detection rate as a function of SDSS $u$ magnitude. Detection rates are shown for the NUV (blue) and FUV (purple) bands. Each redshift bin represents about 5000 quasars observed with GALEX in the given band. Quasars are included only if they have no SDSS neighbour within 10 arcsec.

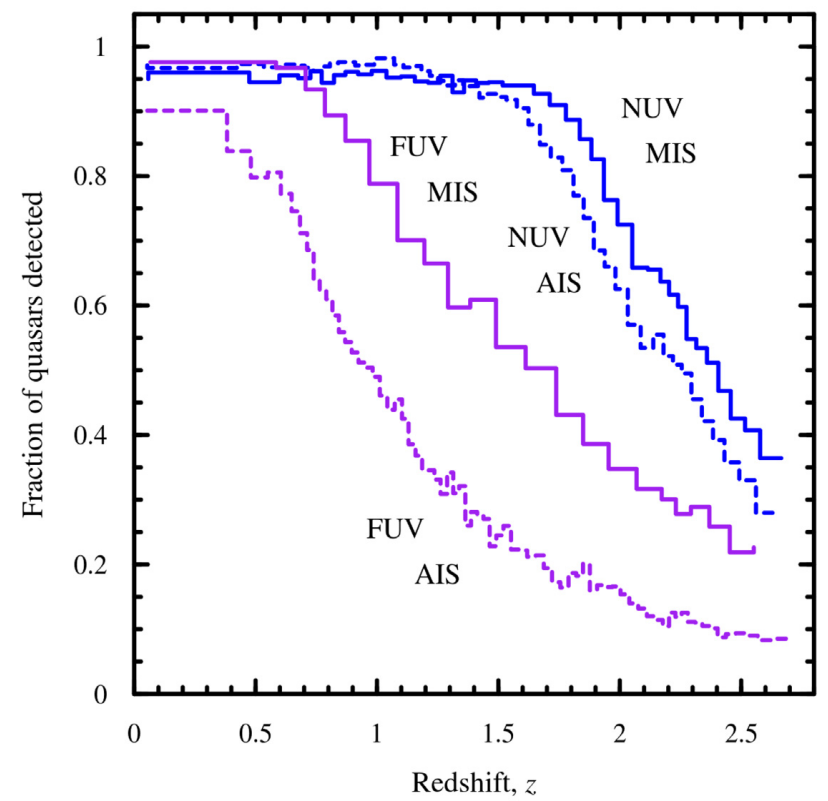

Figure 10. The GALEX quasar detection rate as a function of redshift, for optically bright $(u \leq 21)$ quasars. Detection rates in the NUV (blue) and FUV (purple) bands are shown for both MIS-like (solid) and AIS-like (dashed) exposure times. Each redshift bin represents 1000 quasars observed with GALEX in the given band and exposure time. Too few bright quasars are available for analysis beyond $z \approx 2.7$.

detection fraction for these bright quasars remains above 90 per cent up to a redshift of 1.8 in the NUV band, and up to 0.7 in the FUV band. Beyond those redshifts the detection fraction drops steadily, caused in part by an increasing amount of absorption of quasar light by intervening intergalactic gas. The detection fraction in AIS-like 
images drops to 50 percent at $z \approx 1.0$ and $z \approx 2.3$ in the FUV and NUV bands, respectively. Descriptions of the full range of quasar properties, such as estimates of the magnitude distributions, will generally become increasingly less reliable at redshifts higher than those (see e.g. Section 3).

\subsection{Matched SDSS/GALEX quasar catalogue}

The catalogue of quasar data contains information on 483161 SDSS DR14Q quasars covered by at least one GALEX tile described in the GR6/7 data release. SDSS quasars not observed by GALEX are also included with default values, so that each row corresponds to the same object row in the DR14Q catalogue. The catalogue quantities are listed in Table 1. The information contained in the catalogue allows sub-samples to be selected in exactly the same way as described here. The machine-readable catalogue can be accessed online from the VizieR service.

\section{THE DEPENDENCE OF ULTRAVIOLET QUASAR COLOURS ON REDSHIFT}

The broad-band colours of quasars exhibit distinct patterns with redshift, as strong features common to most quasars shift into and out of the photometric passbands (e.g. Richards et al. 2002). Median colour trends have been used to define relative quasar colours, and study the properties of quasars by intrinsic colour and dust reddening (e.g. Richards et al. 2003, 2002). In this section we examine the EUV colours of the DR14Q quasars, as functions of redshift, at rest wavelengths that in optical studies are either inaccessible, or heavily attenuated by IGM absorption. For clarity, and consistency with the GALEX bandpass notation, we define the EUV region to extend from $\sim 100 \AA$ to the Ly $\alpha$ transition, the FUV region to extend from Ly $\alpha$ to $\sim 2000 \AA$, and the near-UV region to extend from $\sim 2000$ to $\sim 4000 \AA$, at which point the optical region begins.

\subsection{Calculation of colour-redshift curves}

A quasar was selected for the colour analysis if the nearest GALEX neighbour was detected either closer than 3 arcsec, or farther than 7 arcsec from the SDSS position (Section 2.3), no BAL system was identified, no SDSS neighbour was identified within 10 arcsec, and a valid $u$ magnitude exists. As discussed in Section 2.3 , SDSS $u$ magnitudes become unreliable beyond $u \approx$ 22.5, which is the limit we use here.

BALQSOs that are identified in the DR14Q catalogue are omitted here, since their colours are typically not representative of the general quasar population. However, only high-ionization BALQSOs with redshifts greater than about $z=1.5$ have been identified, due to the limited SDSS spectral range over which they can be found. About 8 per cent of the higher redshift quasars are identified BALQSOs. The results of Allen et al. (2011) suggest that this fraction should drop rapidly with decreasing redshift. We show in Section 3.2 that including the known BALQSOs makes very little difference to the median colour values at $z \gtrsim 1.5$. While this suggests the effect of BALQSOs at lower redshift is negligible, little is known about the incidence and properties of low-redshift BALQSOs. We caution that until BALQSOs can be identified over the full redshift range, their effect on the results here will remain unknown.

We apply corrections for Milky Way dust extinction to both the measured UV magnitudes (if they exist), and the magnitude limits. The proper extinction model and reddening values (quantified by
$E(B-V))$ to use in the UV is a matter of some controversy. Most studies have used the reddening values from the maps of Schlegel, Finkbeiner \& Davis (1998, SFD), and their calculated values of the ratio of extinction to reddening in a given band $x$, $R_{x} \equiv A_{x} / E(B-V)$. More recently, various studies have identified systematic offsets in the SFD reddening values. However, some studies (e.g. Schlafly et al. 2010; Schlafly \& Finkbeiner 2011; Sun et al. 2018) find that SFD overestimate the values, while others (e.g. Peek \& Graves 2010; Planck Collaboration XI 2013) find that SFD underestimate the values. Also, the $R_{x}$ values are sometimes found to vary with reddening, especially in the UV (e.g. Peek \& Schiminovich 2013). These discrepancies will not be resolved here. For this study we adopt the extinction correction prescription described by Peek \& Schiminovich (2013), which is based upon a very wide-area extragalactic study in the UV at high Galactic latitudes, using GALEX data. That study showed that standard extinction curves substantially underestimate attenuation in the NUV, and especially FUV bands, and that the $R_{\mathrm{NUV}}$ and $R_{\mathrm{FUV}}$ values depend upon the $E(B-V)$ values given by SFD. For the optical bands, corrections based upon SFD appear to be adequate for quasars (Wolf 2014). Extinction corrections as a function of Galactic location and reddening are still a matter of much study, and are one of the largest sources of uncertainty in the analysis here, especially in the UV. We also note that the final version of the DR14Q catalogue (v4_4) contains only default extinction values (-9999) for about 80000 objects; we have therefore extracted the correct extinction values from the SDSS SkyServer data base. $^{2}$

The initial median FUV - NUV colour-redshift curve for the full sample up to redshift 3, calculated using only the objects detected in both bands, is shown in Fig. 11 (black line). Colourredshift curves are also shown for samples from different ranges of effective exposure time, namely AIS-like (60-400 s), MIS-like (700-3600 s), and very long ( $\left.t_{\mathrm{eff}}>10^{4} \mathrm{~s}\right)$ exposure times. While the overall shapes are similar, the UV colours become dramatically redder with increasing exposure time. This effect is due to the fact that a significant and often large fraction of quasars is undetected in the GALEX bands (Section 2.3). For a given optical magnitude and fixed GALEX exposure time, UV brighter and therefore bluer quasars are more likely to be detected than their UV-redder counterparts. Thus, the sample of detected quasars becomes redder, and presumably more representative, in the UV bands with increasing GALEX exposure time.

This effect is further illustrated in Fig. 12. GALEX observations restricted between $z=0.8$ and $z=1.0$ were grouped into 10 bins of increasing exposure time, each containing $\approx 1000$ detections. The median colours of the detections were calculated and plotted against the detection rate in each bin, which ranges from $\approx 25$ per cent to $\approx 80$ per cent. As the detection rate increases with longer exposure time, the colours become redder, showing that the brightest and most easily detected quasars are clearly UV bluer than their dimmer counterparts. This would strongly bias any colour studies using UV bright quasars as their sample set, since these do not fairly represent the entire quasar population. Unfortunately, restricting the analysis to the longest exposure times severely reduces the sample of observed quasars, and still does not guarantee that the detected objects are representative.

We address these limitations by using survival analysis statistics, which account for the lower magnitude limits in the UV bands

\footnotetext{
${ }^{2}$ skyserver.sdss.org
} 
Table 1. Column description of the catalogue of GALEX GR6/7 UV data for SDSS DR14Q quasars.

\begin{tabular}{lcl}
\hline Column & Name & Description \\
\hline 1 & seqQSO & SDSS DR14Q catalogue sequential row number \\
2 & DR14Q & SDSS DR14Q coordinate-based name, from DR14Q \\
3 & RAdeg & GALEX right ascension (J2000), if matched within 7 arcsec, in degrees \\
4 & DECdeg & GALEX declination (J2000), if matched within 7 arcsec, in degrees \\
5 & Z & Redshift, from DR14Q \\
6 & BAL & Broad absorption line (BAL) flag (Y/N) \\
7 & FUVmag & GALEX FUV magnitude if detected \\
8 & e_FUVmag & GALEX FUV magnitude uncertainty \\
9 & NUVmag & GALEX NUV magnitude, if detected \\
10 & e_NUVmag & GALEX NUV magnitude uncertainty \\
11 & umag & SDSS $u$ magnitude, from DR14Q \\
12 & e_umag & SDSS $u$ magnitude uncertainty \\
13 & l_FUVmag & GALEX FUV detection limit \\
14 & 1_NUVmag & GALEX NUV detection limit \\
15 & sepCoord & Separation between SDSS and GALEX coordinates, if matched within 7 arcsec, in arcsec \\
16 & dCent & Distance of SDSS coordinate from $G A L E X$ tile centre, in arcmin \\
17 & tile_FUV & GALEX FUV tile number \\
18 & tile_NUV & GALEX NUV tile number \\
19 & tEff_FUV & GALEX FUV effective exposure time, in seconds \\
20 & tEff_NUV & GALEX NUV effective exposure time, in seconds \\
21 & numNbr15 & Number of SDSS imaging neighbours out to 15 arcsec \\
22 & dNNbr15 & Distance to nearest SDSS imaging neighbour, if within 15 arcsec, in arcsec \\
23 & uNbr15 & SDSS $u$ magnitude of brightest SDSS imaging neighbour within 15 arcsec \\
\hline
\end{tabular}

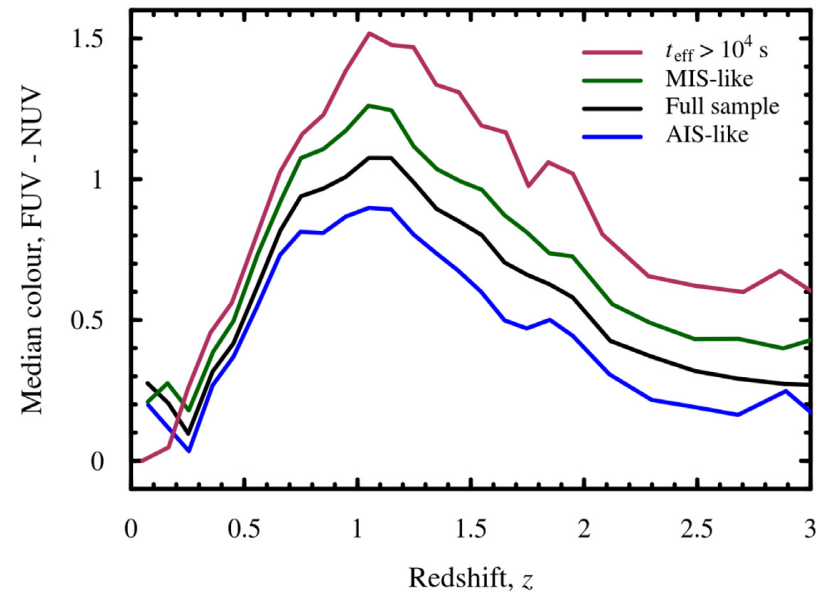

Figure 11. GALEX FUV - NUV median colour as a function of redshift, for the full sample (black line), and for samples observed at different ranges of effective exposure times. Colours for the AIS-like exposure times (60$400 \mathrm{~s})$ are in blue, for the MIS-like exposure times (700-3600 s) are in green, and for very long exposure times $\left(t_{\mathrm{eff}}>10^{4} \mathrm{~s}\right)$ are in maroon. Only detected objects have been used for each curve. No accounting has been made for observed, but non-detected objects.

(Section 2.2.5). The distribution of NUV $-u$ colours is shown in Fig. 13 for a set of about 10000 quasars with redshifts near $z \approx$ 1.5 , which is near the median DR14Q redshift, and where Lymanlimit systems (LLSs) reach the middle of the NUV band. The distribution of detected quasars (black) is slightly skewed to redder colours. However, about 21 per cent of the quasars are undetected in the NUV band, and their colour limit distribution (red) shows that the true colour distribution must be skewed much more heavily to redder colours. The redward skew of the colour distribution is present at nearly all redshifts to varying degree, generally increasing with redshift. The results are similar for the FUV $-u$ colours.

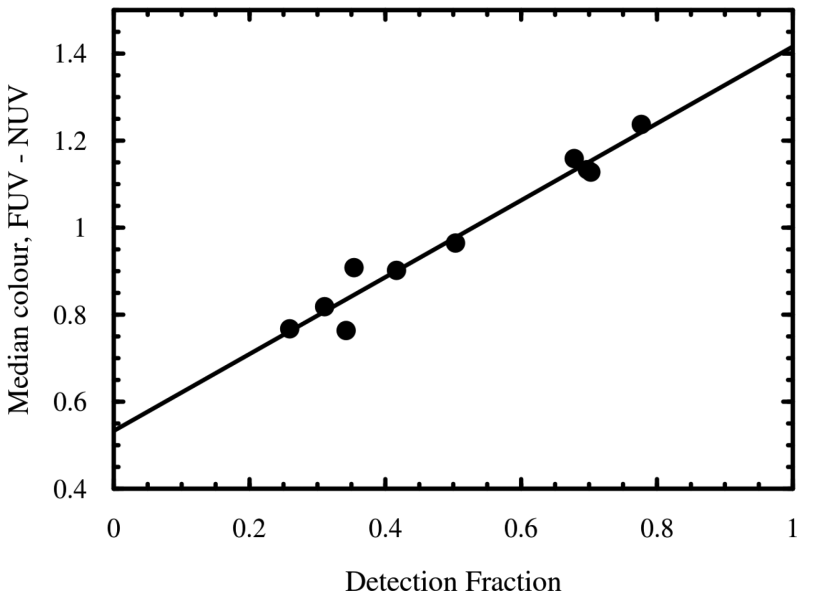

Figure 12. GALEX FUV - NUV median colour of detected quasars, for $0.8<z<1.0$, as a function of detection fraction. Each point represents $\sim 1000$ GALEX detections from which the median colour is calculated, with uncertainty comparable to the point size. The trend is well described by a line fit which shows the colours clearly becoming redder with increasing detection fraction.

Statistical quantities of the colour distributions must be determined by properly accounting for the lower colour limits. This is approached here in two ways. First, quantities such as the median colour are determined from the Kaplan-Meier estimate (KME) of the survival function (Kaplan \& Meier 1958). The KME is widely used in the analysis of censored astronomical data (e.g. Feigelson \& Nelson 1985). However, summary statistics based upon the KME may be biased (e.g. Chen, Hollander \& Langberg 1982; Zhong \& Hess 2009; Khan \& Shaw 2016), in particular when the censoring fraction is high, or when the distribution is heavily skewed. Also in such cases, the estimated survival curve may not extend far enough to measure the median value of the distribution. 


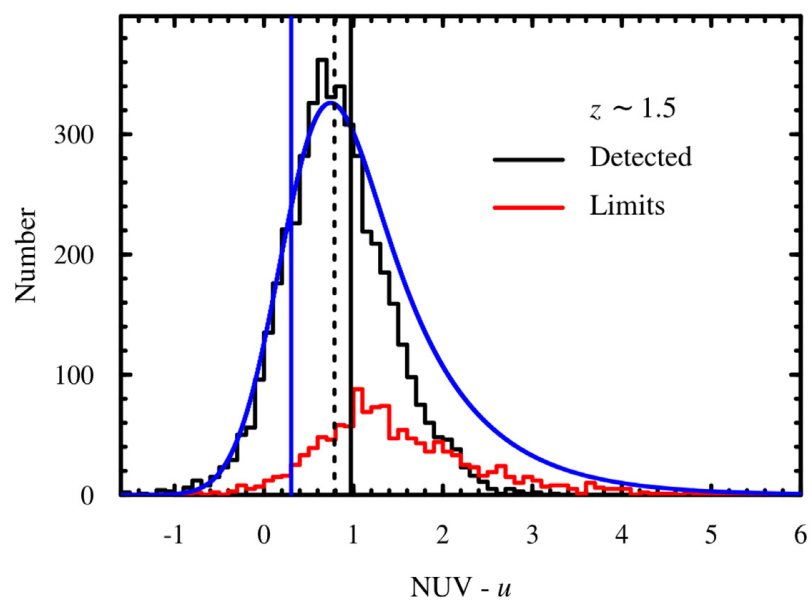

Figure 13. Distribution of NUV $-u$ colours for quasars near $z=1.5$. The black histogram shows the colours of the quasars detected in the NUV band, while the red histogram shows the lower colour limits of the undetected quasars. The detected fraction is just under 79 per cent. The blue line is the maximum likelihood fit of an EMG distribution to the colours, including the limits. The solid black vertical line shows the median value of the EMG distribution. The dotted vertical line shows the median colour of only the detected quasars. The blue line shows the EMG $\mu$ value, which is the centre of the non-convolved Gaussian distribution.

A second approach taken here to account for colour limits is to fit a parametrized distribution curve to the colours using maximum-likelihood estimation (MLE). We have found that an exponentially modified Gaussian (EMG) function works well at most redshifts. The EMG is a three-parameter distribution function that is the convolution of the Gaussian and exponential probability density functions, which results in a skewed distribution (to redder colours in this case). This form may be plausibly expected if the intrinsic colours are drawn from a Gaussian distribution, and are reddened by varying amounts of dust or intergalactic absorption (although other explanations are possible). The EMG is commonly parametrized as

$$
\begin{aligned}
f_{\mathrm{EMG}}=\frac{A \sigma}{\tau} & \sqrt{\frac{\pi}{2}} \exp \left(\frac{1}{2}\left(\frac{\sigma}{\tau}\right)^{2}-\frac{x-\mu}{\tau}\right) \\
& \times \operatorname{erfc}\left(\frac{1}{\sqrt{2}}\left(\frac{\sigma}{\tau}-\frac{x-\mu}{\sigma}\right)\right),
\end{aligned}
$$

where $A$ is the amplitude, $\mu$ is the Gaussian mean, $\sigma$ is the Gaussian standard deviation, $\tau$ is the exponential scale, and erfc is the complementary error function (Kalambert et al. 2011); here $x$ represents quasar colour. The logarithm of the likelihood of obtaining a given set of colours is then (modulo an additive constant),

$$
\log L=\sum_{n} \log f_{\mathrm{EMG}}+\sum_{m} \log \left(1-F_{\mathrm{EMG}}\right),
$$

where $n$ is the number of detected colours, and $F_{\text {EMG }}$ is the cumulative distribution function of the EMG, which accounts for the probabilities of the $m$ lower colour limits. The values of the distribution parameters are found by maximizing this function. An example EMG fit to a set of colours in a narrow redshift range near $z=1.5$ is shown in Fig. 13. The median value of the fitted distribution is significantly redder than the median of only the detected colours. The $\mu$ parameter value, which is the mean of

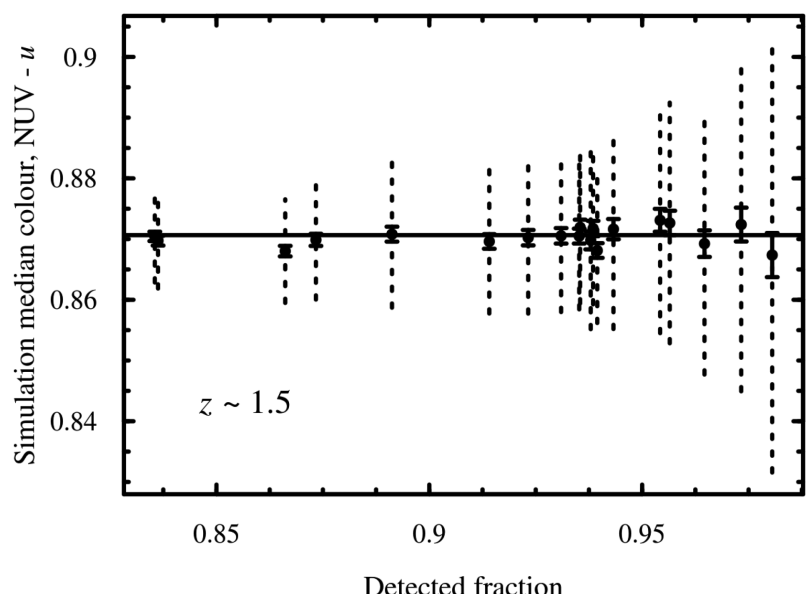

Figure 14. Simulation median NUV $-u$ colour versus NUV detected fraction, at a quasar redshift of about 1.5 (where LLS edges reach the middle of the NUV band). The dots show the average values at various detection fractions, corresponding to different limiting effective exposure time values. The solid error bars show the standard error of the mean for each set of simulations, and the dotted bars show the standard deviation of the simulation values. The simulation averages are consistent with the median colour value of the reference sample, shown with a horizontal line. The standard deviation of the simulation values increases with detection fraction, showing that there is no advantage to restricting the analysis to longer effective exposure times.

the non-convolved Gaussian component, is substantially bluer than either median value.

To check for biases and assess the uncertainties of the results of the KME and MLE methods, we have applied the methods to simulated data sets with known EMG distributions and censoring patterns, similar to those in the real data set. At a given redshift, the colour censoring pattern strongly depends upon the set of $G A L E X$ effective exposure times, $t_{\text {eff }}$. Selecting a sub-sample with a large $t_{\text {eff }}$ cut-off limit will increase the detected fraction (lower the censored fraction), but at the expense of reducing the size of the sample. To test the NUV $-u$ fit dependence on $t_{\mathrm{eff}}$ and censoring fraction, a reference sample from a narrow redshift range was drawn from the real data, and its colour distribution fit with an EMG function using the MLE technique. A lower $t_{\text {eff }}$ value was then imposed upon the reference sample, leaving a truncated subset of $N$ objects.

The simulated sample was constructed by first drawing randomly, with replacement, $N t_{\text {eff }}$ values from the truncated sample, and $N u$ magnitude values from the full reference sample (assuming $t_{\text {eff }}$ and $u$ are independent). NUV magnitudes were assigned to the simulated sample, by drawing randomly from the fitted colour distribution, and adding the respective $u$ magnitudes to the colours. For a given object, the assigned NUV value was either detectable, or a lower limit was assigned (according to equation 5), depending on the assigned $t_{\text {eff }}$ value. The resulting set of NUV $-u$ colours and limits was then fit with an EMG function using the MLE technique. This process was repeated a large number of times at a range of $t_{\text {eff }}$ cut-off limits.

Fig. 14 shows the average, standard error, and standard deviation of the fitted median NUV $-u$ colours of the simulated samples, for a reference sample near $z=1.5$. The colours are shown as a function of the NUV-detected fraction in each set of simulations. The detected fraction is always greater for larger cut-off $t_{\text {eff }}$ values. The horizontal line shows the fitted median colour value of the 


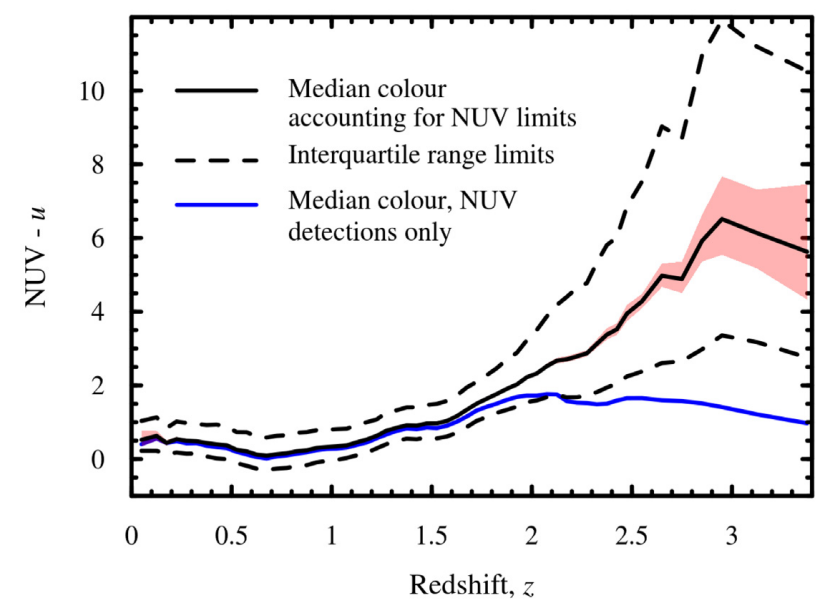

Figure 15. Median NUV $-u$ colour versus redshift, accounting for NUV detection limits (solid black), and using only quasars detected in the NUV band (solid blue). The light red envelope shows the 95 per cent confidence range on the corrected median colour values, based upon a large number of bootstrap samples. The confidence range becomes too large for reliable colour estimates beyond $z \approx 3.4$. The dashed lines show the interquartile range of the full colour distribution based on the EMG fit.

reference sample, which is consistent with the standard errors (solid error bars) of the simulated colours. The results show no evidence for bias when using the MLE technique. It is also evident that the dispersion of the median colours (standard deviation shown as dashed lines) is smaller at lower detection fractions. This means that the results are more precise when larger numbers of objects are analysed, or when the cut-off $t_{\mathrm{eff}}$ is smaller. Similar results are found at different redshifts, and for the FUV $-u$ colours. Therefore, in the following analysis no cut-off $t_{\text {eff }}$ is imposed on the samples.

\subsection{Quasar colour-redshift relationships}

The NUV $-u$ versus redshift relation is shown in Fig. 15. The solid black line shows the median NUV $-u$ values calculated using the MLE technique. The light-red envelope shows the 95 percent uncertainty range of the median colour, calculated from the results of 1000 bootstrap resampled simulated data sets, drawn from the real data set. Beyond a redshift of $\approx 3.4$ the uncertainty becomes very large ( $\gtrsim 1$ mag.), and the results are unreliable. The KME median values are nearly identical with the MLE values, except that the KME survival curve does not reach the median value at redshifts beyond $z \approx 2.6$, so the median values there are undefined. The dotted lines show the interquartile range of the colour distribution, taken from the EMG fit to the data, including NUV detection limits. The interquartile range is asymmetric around the median values, showing the colour distribution is skewed redward.

The blue line in Fig. 15 shows the median NUV $-u$ colour calculated using only the objects with NUV detections. The detected-only median colours are bluer than the MLE colours at all redshifts, and the curves start to diverge strongly at $z \approx 1.5$. The reason for this is that for a given $u$-band magnitude, bluer objects are more likely to be detected than red objects in the NUV band. The sample of NUV-detected objects selectively omits a large fraction of redder objects, causing the median colour estimate to be biased towards bluer quasars. The fraction of objects omitted increases with redshift, as the colour distribution becomes redder. It is clear from this comparison that the colour limits of the undetected objects

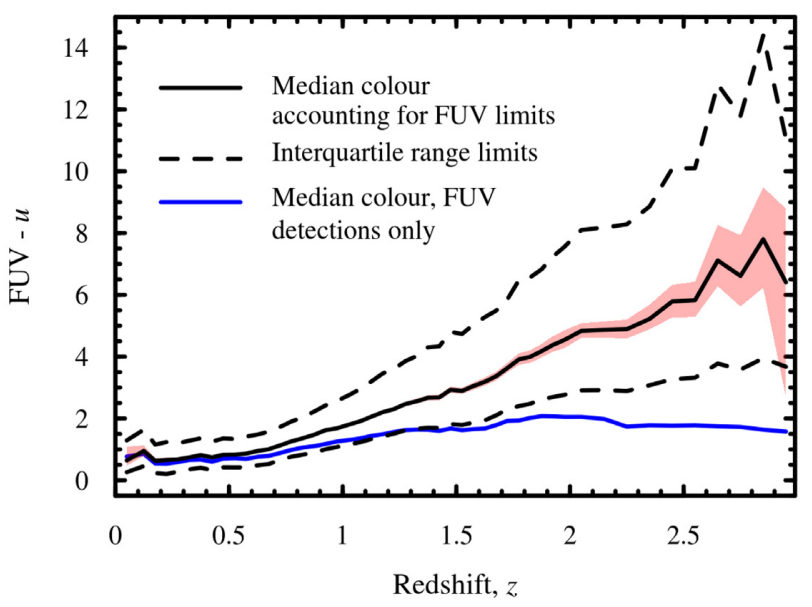

Figure 16. Same as Fig. 15, but for the FUV $-u$ colour versus redshift. The confidence range (light red) becomes too large for reliable colour estimates beyond $z \approx 3.0$.

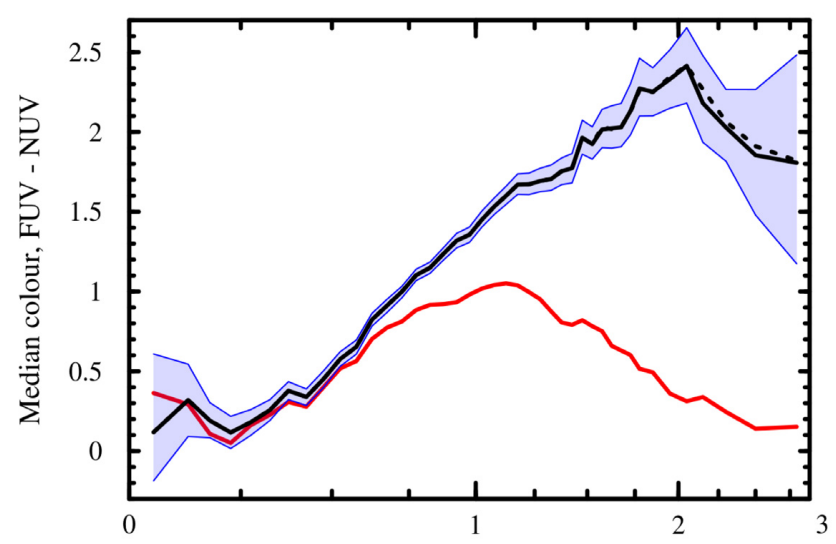

Redshift, $z$

Figure 17. Median FUV - NUV colour versus redshift, accounting for detection limits in both bands (solid black), using reference to $u$-band detections. The median colour-redshift curve constructed only with quasars having detections in both UV bands is also shown (red). The blue envelope shows the 95 per cent confidence range on the corrected colour values, based upon a large number of bootstrap samples. The confidence range becomes too large for reliable colour estimates beyond $z \approx 2.8$. The dashed line shows the colour values when known BALQSOs are included in the sample.

need to be taken into account for accurate estimation of the median colours.

The colour-redshift relationship for the FUV $-u$ colours is shown in Fig. 16. The median colour curve, median uncertainty range, interquartile range, and detected-only curve were constructed in the same way as for the NUV $-u$ curves. In this case, the uncertainty becomes unusably large at $z \approx 3.0$. Again, the detectedonly curve is substantially bluer than the MLE curve, and the colour becomes generally redder with increasing redshift.

A FUV - NUV curve cannot be constructed in the same way as the NUV $-u$ and FUV $-u$ curves, since in many cases the quasars are detected in neither the FUV nor NUV bands. Instead, the FUV - NUV colour curve was estimated by subtracting the NUV $-u$ colour curve from the FUV $-u$ curve, since nearly all of the quasars have $u$-band detections. The results are shown in Fig. 17. The black line shows the median colour for the real data. The 
light-blue band shows the 95 per cent uncertainty range, determined from the results of 1000 bootstrap resampled simulated data sets, drawn from the real data. The red line shows the median colour relation considering only the quasars detected in both UV bands. The detected-only colours are consistent with the MLE colours only up to a redshift of $z \approx 0.4$, at which point the two curves begin to diverge quickly.

BALQSOs identified in DR14Q were omitted from the analysis. However, as discussed in Section 3.1, BALQSOs at $z \leq 1.5$ cannot be identified. The dashed line in Fig. 17 shows the colour-redshift relationship when known BALQSOs (all at $z>1.5$ ) are included in the sample. The colour values are only slightly redder than those of the cleaned sample, if at all, and are well within the median colour uncertainty. This small difference, along with the likely smaller fraction of BALQSOs at lower redshifts (Allen et al. 2011), suggests that the unavoidable inclusion of BALQSOs at $z<1.5$ has negligible effect. We caution, however, that very little is known about BALQSOs at low redshifts, and their influence on the colours here remains uncertain.

\subsection{Quasar template fitting to UV colour-redshift relationship}

Quasar colour-redshift trends are the result of a number of factors, including quasar continuum and emission spectra, absorption and extinction due to intrinsic gas and dust, and absorption and extinction due to intervening systems and the IGM. In this section, quasar spectral models are constructed to determine whether any of these factors, or a combination, can account for the observed colourredshift trends. We construct template spectra based upon UV and optical composites, and compute synthetic GALEX colours to see what variations are consistent with the observed colour-redshift trends.

The primary quasar template spectrum consists of a combination of the composite quasar spectrum constructed by Stevans et al. (2014) from HST/COS spectra, and the SDSS composite of Vanden Berk et al. (2001), which together cover the EUV through optical rest frame. The COS composite was generated from spectra of 159 AGNs, cleaned of narrow absorption lines, and corrected for LLS absorption. The composite covers a rest wavelength range from 475 to $1875 \AA$, although fewer than 10 quasars contribute at wavelengths shortward of $600 \AA$. The SDSS composite was generated from optical spectra of over 2200 quasars, covering a rest wavelength range of 800 to $8550 \AA$. The SDSS composite is not corrected for absorption shortward of the Ly $\alpha$ emission line.

The combined template spectrum was made by first removing the fitted power-law continua described in each paper, resulting in largely continuum-free emission spectra. The emission spectra were scaled to have identical integrated flux between 1300 and $1745 \AA$. The scaled emission spectra were cut and joined at $1745 \AA$, at which point the flux densities matched nearly exactly. The COS and SDSS spectra were used shortward and longward of $1745 \AA$, respectively. Power-law continuum spectra were added in the FUV and EUV regions. A continuum with a power-law slope of $\alpha_{v}$, FUV $=$ -0.46 was added between 1102 and $4212 \AA$, scaled so that the spectrum longward of $1745 \AA$ A exactly matched the SDSS composite. Shortward of $1102 \AA$ a power law with a slope of $\alpha_{v}$, EUV $=-1.41$ was joined to the FUV continuum, to match the EUV slope of the COS spectrum found by Stevans et al. (2014). Additional continua were added at wavelengths longward of $4212 \AA$, but those are irrelevant since the GALEX bands do not cover those regions. The template spectrum covers both GALEX passbands up to a redshift of about 1.8. At wavelengths shorter than $475 \AA$, the EUV power-law

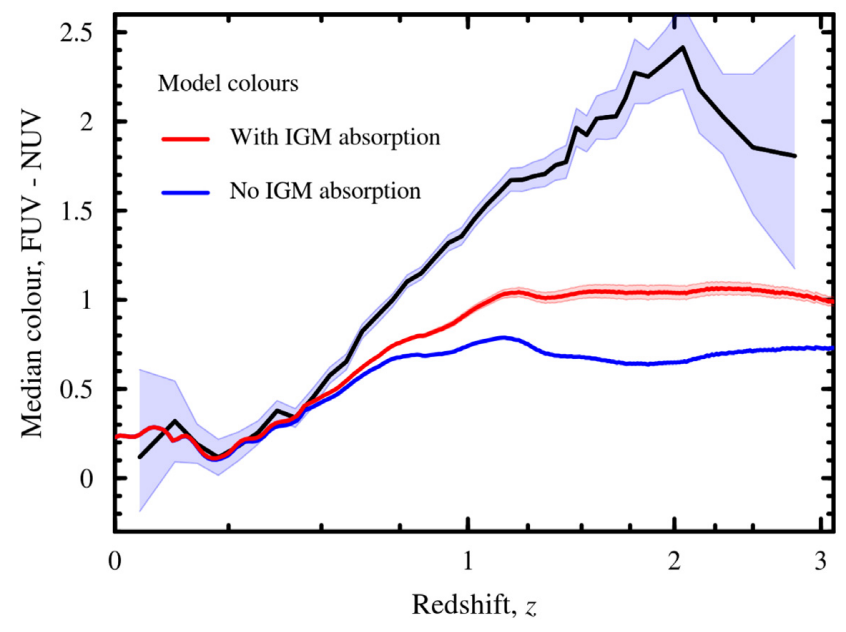

Figure 18. Median quasar FUV - NUV versus redshift (black line and light blue uncertainty envelope) compared to model colours for the default template. Template colour trends are shown without IGM absorption (blue), and accounting for average IGM absorption (red). The range of colours that occur with a 10 percent variation in the IGM opacity is shown with the light red envelope. The redshift scale is stretched logarithmically in $1+z$ to provide more detail at lower redshifts.

continuum is simply extended as a crude model to compute colours at higher redshifts.

The resulting template spectrum is easily modified by changing the power-law continuum slopes, changing the relative scale of the emission to continuum flux densities, including absorption and extinction features, and varying the redshift. Synthetic colours were calculated from modified spectra using the GALEX passband sensitivity functions of Morrissey et al. (2007). The synthetic FUV - NUV colour versus redshift for the unmodified template spectrum is shown by the blue line in Fig. 18. The redshift axis is shown on a logarithmic $(1+z)$ scale to emphasize the details at lower redshifts. The template colours track the measured colour trend remarkably well up to redshifts of about 0.4 , at which point the template colours become far too blue. The template is modified in various ways to see what may explain the increasingly redder measured colours.

The first test is to determine how much of the increasing reddening trend in the UV bands can be attributed to IGM attenuation. At high redshifts, absorption by Lyman series transitions, including LLSs severely attenuate quasar flux shortward of the Ly $\alpha$ line, causing colours that sample both sides of the line to become generally redder with redshift. It is not obvious how the IGM affects UV colours, since the Ly $\alpha$ line enters the NUV band at $z \approx 0.4$ and leaves it at $z \approx 1.4$. Also, the attenuation is much smaller at lower redshifts. IGM attenuation was applied to the template spectrum according to the analytic prescription of Inoue et al. (2014), who account for the distribution and redshift evolution of the Ly $\alpha$ forest, LLSs, and damped Ly $\alpha$ systems, including available low-redshift data. The resulting colour-redshift trend is shown by the red line in Fig. 18. It is clear that IGM absorption tends to redden the FUV - NUV colour, especially beyond redshifts of about 0.5 . However, the mean IGM reddening is not enough to account for the colour difference between the GALEX data and the unmodified template.

Recently Deharveng et al. (2019) attempted to deduce the lowredshift $(z \lesssim 1.5)$ IGM opacity using the GALEX colours of a large $(\approx 9000$-object $)$ sample of quasars. They modelled the EUV continuum below $1000 \AA$ as a power law, and varied several parameters for the number density and column density distributions 
of LLSs. (They did not attempt to account for non-detected objects.) They found it difficult to reconcile the observed number densities of LLSs and the EUV slopes of previous studies, such that either a much higher number density, or much redder slope are required. Our study would seem to resolve this tension, by assuming the observed IGM properties at low redshift, and adopting a very red EUV continuum (see later this section). In fact, an extrapolation of fig. 11 of Deharveng et al. (2019) to the observed LLS number density, yields a power-law slope consistent with what we find here. We discuss this comparison further in Section 5. In the fitting analysis here, we use the IGM prescription of Inoue et al. (2014) as given, and allow the EUV slope of our model to vary. According to Fig. 18, it is unlikely that varying the IGM model would realistically bring the template and data into agreement.

Even given the observed IGM properties, Lusso et al. (2015) found that stochastic variations of the IGM absorption, particularly due to the presence or absence of LLSs, could cause fluctuations of $\sim 16$ per cent in the corrected flux density of their $z \approx 2.4$ quasar stacked spectrum. The maximum IGM mean optical depth in their study is $\langle\tau\rangle \approx 1.6$. Thus, the fluctuation of the flux density corresponds to a relative uncertainty of about 10 per cent in the mean optical depth. To see the effect this would have on the UV colours of our template quasar spectrum, we have included a 10 percent variation in the mean optical depth values generated by the Inoue et al. (2014) model. The light red band in Fig. 18 shows the range of colours produced by this variation. The colours vary by no more than about 0.1 magnitude due to uncertainties in the IGM $\langle\tau\rangle$ values. Therefore, while IGM attenuation causes quasar UV colours to be redder, it does not appear to be enough to redden the template spectrum to the observed colour trend at redshifts beyond $\approx 0.5$. In the following analysis, the mean IGM attenuation is included for all modifications of the quasar template spectrum.

Next, the effect of intrinsic dust reddening on the quasar colours is examined. Richards et al. (2003) showed that quasars selected by the SDSS are largely unreddened by intrinsic dust, and that less than about 10 percent are reddened by SMC-like dust with $E(B-V)$ more than 0.04 (see also Hopkins et al. 2004; Krawczyk et al. 2015, for similar conclusions). However, dust extinction preferentially affects shorter wavelength light, so we might expect to observe a greater effect in the far and extreme UV. We adopt an SMClike dust extinction model, since it appears to be consistent with most SDSS quasar reddening (Richards et al. 2003; Hopkins et al. 2004; Hjorth et al. 2013; Krawczyk et al. 2015). However, no observationally derived SMC extinction curve exists that covers the short rest wavelengths observed in the quasar sample. For example, the farthest reaching SMC extinction curves based upon FUSE data extend to only about $1000 \AA$ (Cartledge et al. 2005), which reaches the GALEX FUV band at $z \approx 0.4$. Instead, we use the model extinction curve presented by Weingartner \& Draine (2001), which is derived from inferred grain size distributions in the SMC, and which extends well below the Lyman limit. The model dust attenuation then depends upon the adopted extinction curve, and the value of the colour excess $E(B-V)$. Given the lack of observational extinction curves in the EUV, we caution that SMC-like reddening is not necessarily valid, nor the only possibility in this region (see the discussion in Section 5).

Differing amounts of intrinsic dust reddening, quantified by $E(B$ $-V$ ), were applied to the template quasar spectrum, and the FUV NUV colours were calculated as a function of redshift. Average IGM attenuation was also applied. Fig. 19 shows the resulting colourredshift curves for values of $E(B-V)$ ranging from 0 to 0.05 . At low redshifts, the curves are consistent with little or no reddening,

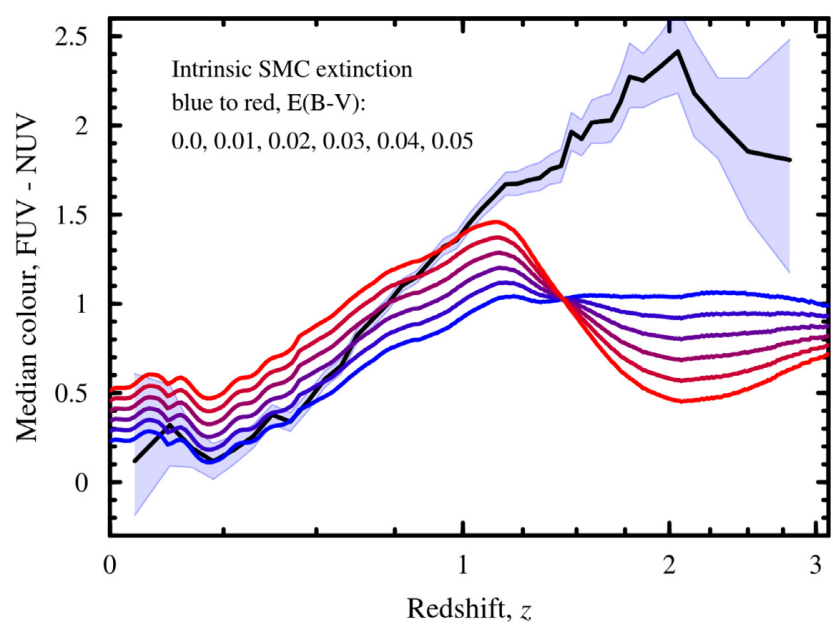

Figure 19. Median quasar FUV - NUV versus redshift (black line and light blue uncertainty envelope) compared to model colours that include intrinsic SMC-like dust reddening. The results of applying extinction to the default template with a range of $E(B-V)$ values, are shown by the coloured curves as indicated. Mean IGM absorption was also applied in the models.

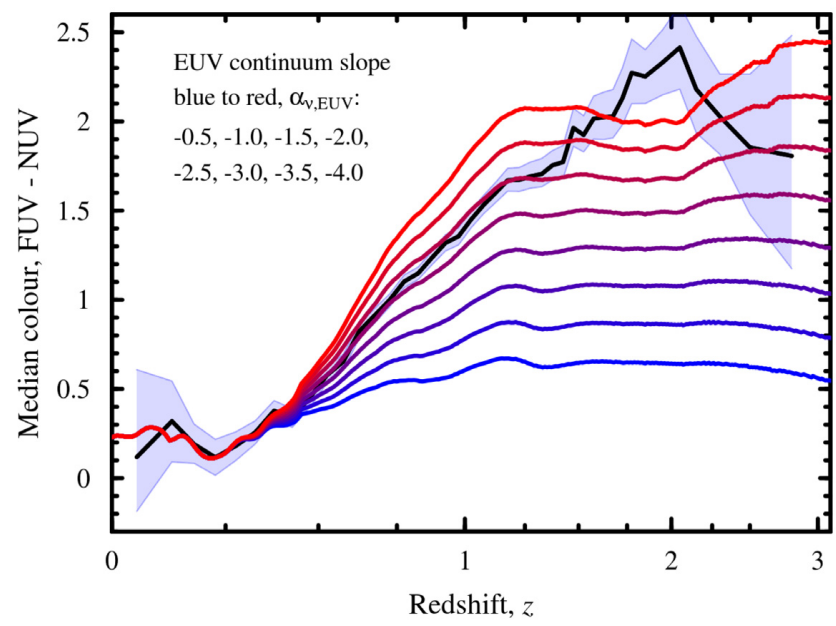

Figure 20. Median quasar FUV - NUV versus redshift (black line and light blue uncertainty envelope) compared to model colours of template spectra with varying EUV power-law slope, $\alpha_{v}$, EUV. The values of the slope range from relatively blue $(-0.5)$ to very red $(-4.0)$, and the UV colours are affected as indicated. Mean IGM absorption was also applied in the models (but no intrinsic dust reddening).

and larger $E(B-V)$ values produce colours that are far too red. At redshifts beyond $\sim 0.7$ all of the colour curves become too blue compared with the data. For intrinsic dust reddening to account for the colour-redshift curve, the model extinction curve would have to be drastically altered, or the dust properties near quasars would have to evolve dramatically.

A third explanation for the steep reddening of the UV colours with redshift is an intrinsically red average quasar continuum in the EUV. We have tested this possibility with a very simple modification to the quasar template spectrum - the EUV continuum slope $\alpha_{v}$, EUV, blueward of $1100 \AA$ was varied and the resulting colour-redshift values were calculated. Fig. 20 shows how the colour-redshift curve is affected by variations in $\alpha_{v}$, EUv ranging from -0.5 to -4.0 . The unmodified template spectrum has $\alpha_{v}$, EUV $=-1.41$ (consistent with Stevans et al. (2014)). The low-redshift colours are still in excellent 


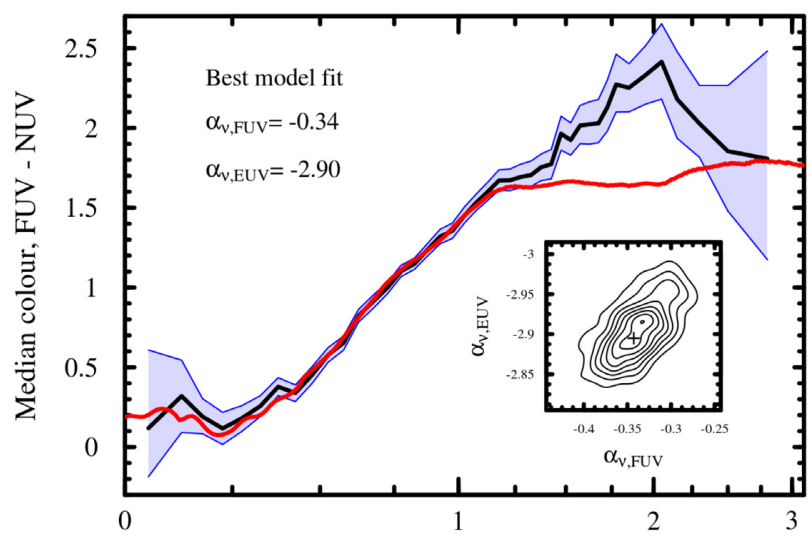

Redshift, $z$

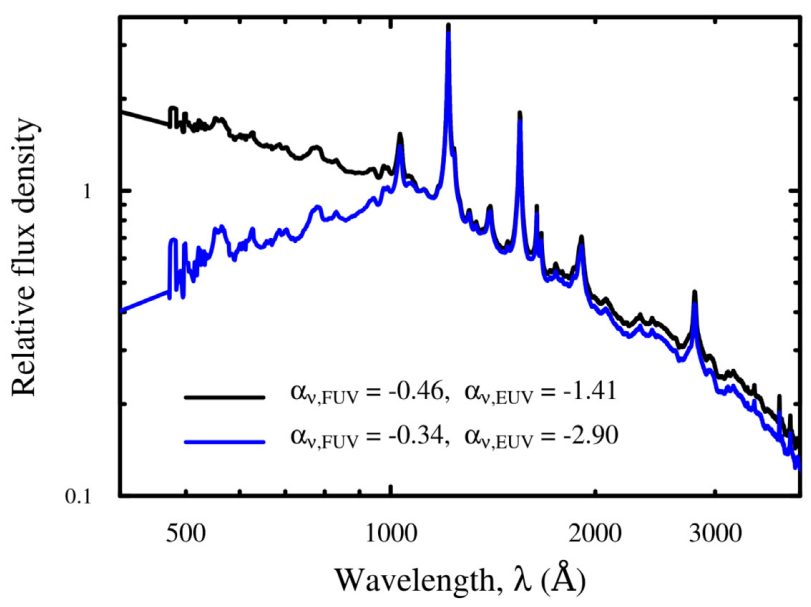

Figure 21. Median quasar FUV - NUV versus redshift (black line and light blue uncertainty envelope), and the best-fitting model (red) that includes IGM absorption, intrinsic dust reddening, and variations to the power-law slopes in the FUV, $\alpha_{v}$, FUV, and EUV, $\alpha_{\nu}$, EUV. The values of the powerlaw slopes for the best-fitting model are given. The inset plot shows the range of slope values for a large number of bootstrap samples. The fit was made with data covering redshifts up to 1.2 ; beyond this the rest-frame template spectrum is highly uncertain. The best-fitting model was extended to redshifts beyond 1.2, by simply extending the EUV power law of the template continuum.

agreement with the data, largely because the EUV spectral change does not reach the middle of the FUV band until $z \approx 0.35$. At higher redshifts, values of $\alpha_{v}$, EUv from about -3.0 to -3.5 produce colours that appear to track the curve reasonably well.

Each of the factors examined so far - IGM attenuation, intrinsic dust reddening, and EUV spectral slope variations - can contribute significantly to explaining the UV colour-redshift curve. We fit a model that includes these factors, and also variations in the FUV spectral slope, to the observed curve. The model has three adjustable parameters: the EUV spectral slope $\alpha_{\nu \text {, EUV }}$, the FUV spectral slope $\alpha_{v, \mathrm{FUV}}$, and the colour excess of intrinsic extinction $E(B-V)$. The average IGM attenuation as a function of redshift is assumed, as prescribed by Inoue et al. (2014). The transition wavelength between the extreme and FUV continuum is fixed at $1102 \AA$, consistent with Stevans et al. (2014). The intrinsic SMC reddening curve extending to short wavelengths is from Weingartner \& Draine (2001), with a lower limit of $E(B-V)=0$ for the colour excess. In addition, the fit is made only up to a redshift of 1.2 , because the Stevans et al. (2014) composite spectrum is very uncertain shortward of $600 \AA$ (fewer than 10 quasar spectra contribute) which reaches the GALEX FUV band at $z \approx 1.2$. For visual comparison with the data however, the model colours are calculated at higher redshifts by simply extending the template EUV continuum as needed below the limit of the HST/COS composite. The distribution of colourredshift curves from the bootstrap samples is close to Gaussian, so the best fit is evaluated using weighted least squares and the variance of the bootstrap sample results. The variations of the parameter fit values were estimated by fitting the model to a large number of bootstrap samples.

The best-fitting model colour-redshift curve is shown by the red line in Fig. 21. The model is fit only to $z=1.2$, but it is extrapolated to higher redshifts as described above. The best-fitting model has $E(B-V)=0$ (its lowest allowed value), indicating that an intrinsic reddening component is not necessary. The joint distribution of EUV and FUV spectral slopes from the bootstrap
Figure 22. The best-fitting model spectrum (blue), and the default template spectrum (black) for comparison. The FUV and EUV continuum slopes are given for each spectrum. The spectra are normalized to a relative flux density of unity at $1100 \AA$, where the two power laws are joined.

sample fits is shown in the inset of Fig. 21. The best-fitting values of the continuum slopes are $\alpha_{\nu \text {, EUV }}=-2.90 \pm 0.04$ and $\alpha_{\nu \text {, FUV }}=$ $-0.34 \pm 0.03$. The FUV slope is consistent with previous studies, but the EUV slope is much softer (redder) than most previously reported values. A detailed comparison with other studies is given in Section 4.

The corresponding modified quasar template spectrum that produces the best-fitting colours is shown in Fig. 22. The unmodified $H S T / C O S+$ SDSS template spectrum is also shown for comparison. The steep decline of the EUV continuum shortward of $1100 \AA$ is clear in the best-fitting model. It is remarkable that the spectrum produces the colours as well as it does, considering that the model has only three free parameters (or only two in effect, since intrinsic reddening is absent). Other spectral shapes that do at least as well are possible. For example, the EUV to FUV continuum break point could be adjusted, or additional break points could be introduced. However, these modifications would increase the number of free parameters in the model without justification. The colour curve at higher redshifts could be used to distinguish among other models, but this would require information about quasar emission spectra at wavelengths shortward of $\approx 500 \AA$, which is currently lacking.

\section{COMPARISON WITH OTHER UV QUASAR STUDIES}

The model spectrum that describes the DR14Q GALEX colourredshift curve, is much redder in the EUV than the composite spectra of any other UV study. One possible explanation is that space-based spectroscopic studies of quasars are generally biased towards objects that are bright in the rest-frame UV, which in turn may bias the statistical inferences from those studies. For example, the average UV continuum of optically selected quasars in those studies may be biased blueward, if UV-fainter and therefore redder quasars are systematically omitted. In fact, only a small fraction of EUV slopes of individual quasars in those studies (Telfer et al. 2002; Scott et al. 2004; Stevans et al. 2014) is consistent with the value we find here. In this section, we examine whether the GALEX colour distribution of quasars selected to construct spacebased UV composites, can account for the EUV slope discrepancy found here. 
Table 2. EUV continuum power-law slopes of space-based UV quasar samples. The minimum wavelength of the composite or template spectrum $\lambda_{\min }$, and the instruments used for each study are also listed.

\begin{tabular}{lccl}
\hline$\alpha_{\nu, \text { EUV }}$ & $\lambda_{\min }(\AA)$ & Instrument & Reference \\
\hline$-2.90 \pm 0.04$ & 475 & $G A L E X /$ imaging & This work \\
$-0.72 \pm 0.26$ & 425 & $H S T /$ COS & Tilton et al. (2016) \\
$-1.70 \pm 0.61$ & 600 & $H S T /$ WFC3 & Lusso et al. (2015) \\
$-1.41 \pm 0.15$ & 475 & $H S T /$ COS & Stevans et al. (2014) \\
$-1.41 \pm 0.22$ & 500 & $H S T /$ COS & Shull, Stevans \& Danforth (2012) \\
$-1.91 \pm 0.32$ & 700 & $G A L E X /$ grism & Barger \& Cowie (2010) \\
$-0.56_{-0.28}^{+0.38}$ & 640 & $F U S E$ & Scott et al. (2004) \\
$-1.76 \pm 0.12$ & 350 & $H S T /$ FOS + STIS & Telfer et al. (2002) \\
$-1.96 \pm 0.15$ & 350 & $H S T /$ FOS & Zheng et al. (1997) \\
\hline
\end{tabular}

The measured EUV spectral slopes $\alpha_{v}$, EUV, from nine studies are listed in Table 2 in comparison to the value found here. The $\alpha_{\nu \text {, EUV }}$ values range from -2.90 (reddest, found here) to -0.56 (bluest), and many of the values are formally inconsistent with each other. Most of the values are from spectroscopic studies with HST or FUSE. The study by Barger \& Cowie (2010) used a bright flux-limited sample of emission line selected AGN from GALEX grism spectra. The value given here from that study is the weighted average of the two independent but statistically consistent values found in two redshift ranges, covering $0.65<z<1.25$. The SED study of Krawczyk et al. (2013) extends down to $912 \AA$; no EUV spectral slopes are given, but SEDs are provided in tabular form. While not designed for accurate spectral slopes in the EUV, we measured a spectral slope for their full-sample SED between 912 and $1100 \AA$ of about -1.06 . This value is comparable to some of the bluer values in the other studies. Due to the uncertainty of the Krawczyk et al. (2013) SED in this region, the spectral slope value is omitted from Table 2.

Aside from sample bias, several other explanations have been offered to account for the slope differences among the studies. These include the use of different continuum windows (Tilton et al. 2016), the use of continuum windows that are not truly emission free (Stevans et al. 2014), differences in the methods of correcting for IGM absorption (Lusso et al. 2015), uncertainties in corrections for Galactic extinction (Tilton et al. 2016), and the possible correlation between spectral slope and quasar luminosity (Stevans et al. 2014). All of these likely contribute at some level. Here, we show that sample selection differences, in particular the bias towards quasars with bluer UV to optical colours, can explain the wide range of spectral slope differences.

Fig. 23 shows the GALEX FUV - NUV colours of individual quasars observed in five of the studies listed in Table 2. The quasars were observed with the FUSE satellite (Scott et al. 2004), the $H S T / F O S$ and HST/STIS spectrographs (Telfer et al. 2002), the $H S T / C O S$ spectrograph (Stevans et al. 2014; Tilton et al. 2016), the $H S T / W F C 3$ grism (Lusso et al. 2015), and the GALEX/grism. The sample in the study by Shull et al. (2012) is a subset of the Stevans et al. (2014) sample, and the Zheng et al. (1997) sample is a subset of the Telfer et al. (2002) sample. Lists of the observed quasars are given in all but the Barger \& Cowie (2010), Telfer et al. (2002), and Zheng et al. (1997) papers. For the Telfer et al. (2002) quasars, we followed their stated selection criteria as far as was possible; the resulting list is at least representative of their selected sample.

The quasar lists were cross-matched to objects in the GALEX catalogue, in a way similar to that described in Section 2. Nearly 90 percent of the quasars in the lists were covered by at least one GALEX tile, giving a set of almost 500 observed objects. Assuming all GALEX matches within 7 arcsec, are true detections

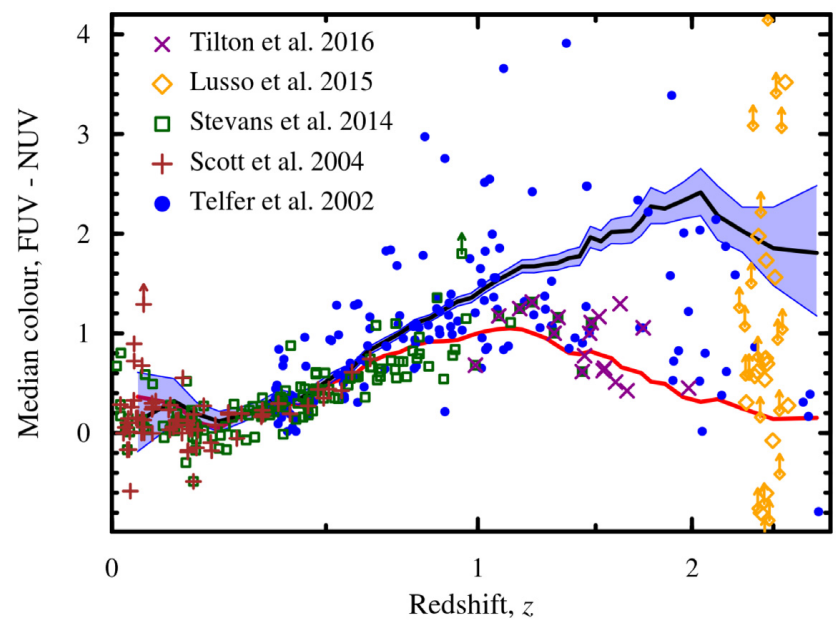

Figure 23. The FUV - NUV colours of quasars used to construct UV composite spectra with space-based observations. Objects used in the listed studies are given with different symbols. Lower limits (when a quasar is not detected in the FUV band) are shown with arrows. The median colour versus redshift curve from this study (black line and light blue uncertainty envelope) is shown for comparison, along with the median colours using only the quasars detected in both GALEX bands (red).

of the quasars, just over 90 per cent and 75 per cent of the quasars were detected in the NUV and FUV bands, respectively. Lower limits on the quasar FUV - NUV colours are given if no detection is found in the FUV band. No colour estimate is possible if an object is detected in neither GALEX band; these cases comprise just under 10 per cent of the total set of objects.

As seen in Fig. 23, the samples tend to cover significantly different redshift ranges, and hence, the GALEX bands cover different rest wavelength ranges in each study. The samples also cover significantly different luminosity ranges, as shown by Lusso et al. (2015). The sample of Scott et al. (2004) has the lowest average redshift, and the lowest average luminosity. The sample of Lusso et al. (2015) has the largest average redshift and average luminosity. Depending on the redshift range, the GALEX colours of the objects tend to be either close to the median values found in this study, or significantly below them (i.e. they tend to be bluer than in this study). In fact, the quasars trace the colour trend of the $G A L E X$ detected-only sample more closely. Only the Telfer et al. (2002) sample appears to have quasars that may produce average colours similar to those found here. To see this more clearly, Fig. 24 shows the FUV - NUV colour differences between the other UV samples and the GALEX sample median. Even at low redshifts, the 


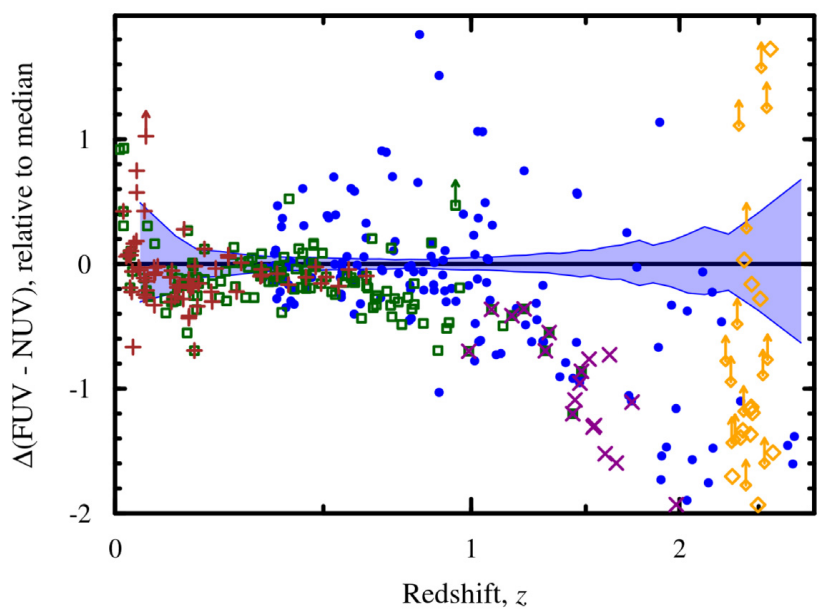

Figure 24. The relative FUV - NUV colours of the quasars used to construct UV composite spectra, compared to the median colour-redshift curve of this study. Symbols are the same as in Fig. 23. Points above the black line are redder than the median values of this study, while points below the line are bluer. The light blue envelope is the uncertainty range from this study around zero relative colour.

average $H S T$ and FUSE colours are bluer by at least several tenths of a magnitude. The difference generally becomes more pronounced at higher redshifts. The reason for this difference is likely that quasar targets for UV spectroscopic studies are typically selected to be bright in the observed UV, relative to their optical brightnesses. This is understandable, since for example, quasar absorption line studies benefit greatly from observing the brightest background sources available. The quasars in studies such as these are therefore likely to be biased towards bluer objects on average.

To test whether the sample colour differences can account for the variation in the measured EUV spectral slopes, synthetic colours were generated for the template quasar spectrum, described in Section 3.3, with varying spectral slope. The template $\alpha_{v}$, Euv value was varied from -4 to just over zero, to cover more than the full range of values listed in Table 2. The synthetic FUV - NUV colours were calculated at five redshifts from 0.5 to 2.4 , covering the mean

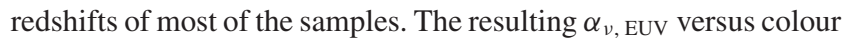
relations are shown in Fig. 25. As expected the colours become redder as the EUV spectral slope becomes more negative (softer). However, the relation is much steeper at smaller redshifts. That is, at small redshifts, a small change in FUV - NUV corresponds to

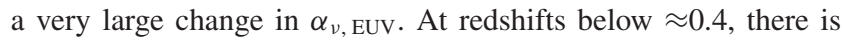

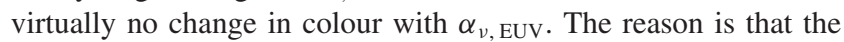
EUV region $(\lesssim 1000 \AA)$ does not even enter the FUV band until $z \approx$ 0.35 . The EUV region covers a larger fraction of the GALEX band widths as redshift increases, and so the colours are increasingly dependent on the EUV spectral slope.

The $\alpha_{v}$, EUv versus colour relationship, coupled with sample selection bias, can explain the range of measured EUV slope values. Fig. 25 shows the measured $\alpha_{v}$, EUv values for the five studies listed in Fig. 23. As mentioned above, the Scott et al. (2004) FUSE sample has the bluest composite EUV slope, and the smallest average redshift. It also has the smallest FUV - NUV colour difference compared to the DR14Q sample (Fig. 24), with colours typically being bluer by a few tenths of a magnitude. But since the redshift of the sample is small, the small colour differences correspond to much bluer $\alpha_{v}$, EUv values. Hence, the FUSE sample turns out to have the

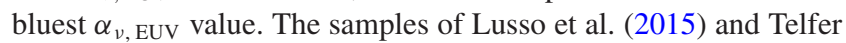

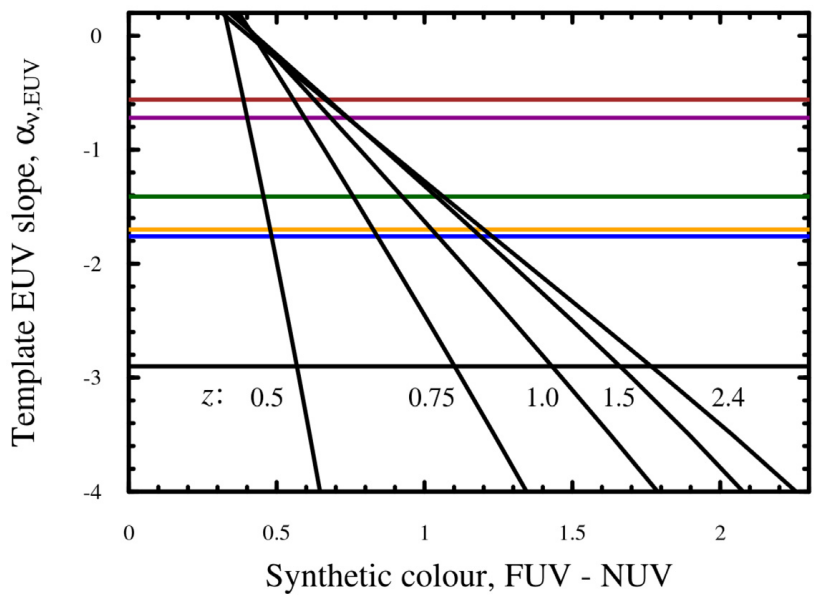

Figure 25. EUV spectral slope of the quasar template, $\alpha_{v}$, EUV, plotted against the resulting synthetic FUV - NUV colour. The slope-colour relation is plotted for five redshifts. The coloured horizontal lines are the measured values of the EUV spectral slopes of composite quasars using FUSE or HST spectra; the colours correspond to the same references as in Fig. 23. The black horizontal line shows the EUV slope of the best-fitting model to the GALEX data. The intersections of the lines at each redshift with the horizontal EUV slope lines show how much the FUV - NUV colours would have to differ to account for differences in the EUV slopes.

et al. (2002) [which includes the Zheng et al. (1997) sample] have the widest distribution of FUV - NUV colours, and come closest to representing the full range of DR14Q colours. These studies also report the reddest composite spectrum $\alpha_{v}$, EUV slopes.

\section{DISCUSSION}

We have shown that the EUV colours of quasars are on average much redder (EUV weaker) than previously found, when a much wider range of quasar brightnesses is considered, and when quasars with UV detection limits are properly accounted for. The spectral models used to match the colour-redshift trends are purely empirical, and not based upon any physical arguments. The power-law continuum models are adopted mainly for their mathematical simplicity and flexibility, and the break points joining the power laws are also arbitrary. While nearly power-law-like continua are expected at optical and near-UV wavelengths in various quasar emission models that simple form is usually not predicted to continue farther into the UV. While a thorough comparison of various quasar emission models with our results is beyond the scope of this paper, here we discuss some of the general expectations of quasar spectral models in the UV, in light of the results from the SDSS+GALEX data.

The workhorse AGN model of accretion from a viscous disc on to a supermassive black hole produces emission spectra in broad agreement with observations (e.g. Shields 1978; Kolykhalov \& Sunyaev 1984; Sun \& Malkan 1989). However, even detailed models suffer from a number of problems when the predicted emission is compared with observed spectra (see the summaries by e.g. Koratkar \& Blaes 1999; Koratkar 1999). In particular, the models are generally too blue in the FUV and EUV, and the continuum peak can vary over a much wider frequency than is observed. Our results are consistent with most previous observations that quasar continua tend to exhibit a break at $\lesssim 1100 \AA$ (e.g. Zheng et al. 1997; Scott et al. 2004; Shang et al. 2005; Stevans et al. 2014; Lusso et al. 2015). Instead, standard geometrically thin, optically thick accretion disc models tend to predict steeper FUV slopes that 
extend well into the EUV before turning over (e.g. Laor \& Netzer 1989; Laor 1990). The results here showing a much weaker EUV spectrum than previous studies exacerbate the discrepancy with those models.

More sophisticated accretion disc models that include some combination of detailed vertical disc structure, relativistic effects, non-LTE effects, continuum opacities of the gas species, and Comptonization (e.g. Ross, Fabian \& Mineshige 1992; Sincell \& Krolik 1998; Hubeny et al. 2000, 2001), improve the agreement with observations, as would be expected. Hubeny et al. (2000) compared some representative fits to the combined composite spectra of Francis et al. (1991) (wavelengths $\gtrsim$ Ly $\alpha$ ) and Zheng et al. (1997) (wavelengths $\lesssim$ Ly $\alpha$ ). The optical to FUV composite is easily fit by a wide range of the combination of black hole mass $M$, and accretion rate $\dot{M}$ (for a fixed black hole spin, viscosity parameter, and inclination angle). However, the EUV region is very sensitive to the precise values of those quantities. For example, reducing the value of $\dot{M} / M^{2}$ by a factor of 2 causes a steep drop in the EUV flux shortward of $\operatorname{Ly} \alpha$, at least qualitatively consistent with what we find here. Such a drop also corresponds to a reduction of luminosity, which may be consistent with the fact that our UV sample is on average less luminous than others. Blaes (2004) fit the Hubeny et al. (2000) model to the combined SDSS (Vanden Berk et al. 2001) and HST (Telfer et al. 2002) composites. The best fit is a good match from the optical to FUV regions, but it drops well below the EUV composite starting just shortward of the O VI / Ly $\beta$ line, again qualitatively consistent with the current results. However, composite spectra have no well-defined physical meaning, and the properties of the quasars used in their construction are expected to vary widely. Model fits to samples of individual spectra (e.g. Shang et al. 2005; Davis, Woo \& Blaes 2007; Shankar et al. 2016) still show significant discrepancies, even in the optical and FUV regions. Thus, while the types of disc models discussed here are promising for explaining the weak EUV flux in the GALEX data, they probably do not yet provide a complete picture.

Another means of reducing the EUV flux in accretion disc models is to invoke mass outflow in the form of a disc wind. Evidence for outflows in AGNs comes from both observations and theoretical considerations (see the review by Crenshaw, Kraemer \& George 2003). The outflows can be driven by temperature, radiation, or magnetic fields. Disc winds tend to make model AGN spectra softer (redder), because they continually remove mass starting from outer disc radii and moving inward, reducing the accretion rate of the hot inner disc where the vast majority of the EUV would originate (Slone \& Netzer 2012; Laor \& Davis 2014; Kazanas 2019). Disc wind models have met with some success at fitting observed AGN spectra in the optical to FUV regions (Capellupo et al. 2015). However, outflow models have yet to be tested with a significant sample of observed EUV continuum spectra.

Aside from variations in accretion disc models, softer quasar spectra could be explained by reddening due to intrinsic or intervening dust, as has often been suggested (e.g. Koratkar \& Blaes 1999; Binette et al. 2005; Davis et al. 2007; Xie et al. 2016). While dust attenuation is undoubtedly present at some level, a number of studies of the SDSS quasars show that only a small fraction $(<10$ per cent $)$ are reddened by more than $E(B-V)=0.04$, with reddening curves consistent with SMC extinction (Richards et al. 2003; Hopkins et al. 2004; Krawczyk et al. 2015). Even in studies of highly reddened quasar sub-samples, SMC-like extinction seems to be consistent with most of the spectra (Maddox et al. 2008; Heintz et al. 2016; Krogager et al. 2016; Wethers et al. 2018). The results of Section 3.3 show that even a relatively small amount of SMC-like dust is inconsistent with the median UV colours at low redshifts. Also, assuming a model extension of the SMC curve into the EUV, dust reddening does not by itself explain the red colours at higher redshifts $(z \gtrsim 1)$.

The very small fraction of heavily dust-reddened quasars suggests that most quasar sightlines contain very little dust (Fynbo et al. 2013). In contrast, some studies have suggested that most quasars are strongly reddened $[E(B-V)$ values $\gtrsim 0.1]$ with substantially flatter extinction curves (e.g. Czerny et al. 2004; Gaskell et al. 2004). Such curves would be difficult to reconcile with the GALEX EUV quasar colours, because they do not attenuate UV light nearly as strongly as the SMC-like curve used here. If intrinsic dust is a major component of most SDSS quasars, it would have to exhibit an extinction curve that is much steeper (removing more blue light), not flatter, at shorter wavelengths than an SMC-like curve. In fact some steeper extinction curves have been reported for small subsamples of quasars, specifically examined for their anomalously red colours (Fynbo et al. 2013; Jiang et al. 2013; Leighly et al. 2014; Zafar et al. 2015; Krogager et al. 2016). Quasars with such curves are scarce, but a large instance of extinction curves steeper than the SMC at EUV wavelengths cannot be ruled out.

Another novel candidate for EUV dust extinction is nanodiamond dust particles (Binette et al. 2005). Theoretical nanodiamond extinction curves have the intriguing property of being strong in the EUV, but relatively weak at NUV and optical wavelengths (Binette et al. 2005; Rai \& Rastogi 2010). While independent evidence for the existence of such dust in AGNs is currently lacking (de Diego et al. 2007), various plausible grain scenarios can be made to fit the EUV turnover observed in a number of individual quasar spectra (Haro-Corzo et al. 2007). Extinction curves such as these cannot yet be ruled out with existing data. In any case, the role of dust in modifying the EUV part of quasar spectra will remain uncertain, at least until realistic extinction curves, of any objects, are observed that extend below $\sim 1000 \AA$.

Extremely red colours could also be accounted for with a grossly different IGM model than adopted here. Realistic stochastic variations of the IGM opacity do not by themselves help the quasar template to approach the red UV quasar colours (Fig. 18). Deharveng et al. (2019) attempted to match a large sample of quasar GALEX colours with simulations in which a power-law EUV continuum and the parameters of a low-redshift IGM model were simultaneously varied. Their results were difficult to bring into agreement with previously published EUV slope and IGM opacity results. However, they considered only models with $\alpha_{v \text {, EUV }} \gtrsim-2.4$. The number density of LLSs in their model is directly correlated with the power-law slope. Assuming this trend in their models can be extended to redder slopes, based upon their Fig. 11, a slope of -2.9 would provide a good match the LLS number density of previous observational studies (Ribaudo, Lehner \& Howk 2011). This agreement supports the conclusion that the low-redshift IGM opacity models are largely correct, and that the red EUV colours we find here are intrinsic to the quasars themselves.

\section{SUMMARY AND CONCLUSIONS}

We have carefully cross-matched the SDSS Data Release 14 quasar catalogue with the final GALEX photometric catalogue, to examine the FUV to EUV colours of a very large sample of quasars. Of the over 480000 SDSS quasars covered by a GALEX tile, nearly 250000 (51 per cent) and 110000 (23 per cent) have highly reliable NUV and FUV detections (true detection probability $>95$ per cent). UV detection limits were determined for all of the quasars without 
GALEX counterparts. The median FUV - NUV versus redshift distribution was calculated using survival analysis to properly account for the non-detections. Forward modelling of a quasar template spectrum, including IGM opacity, intrinsic dust reddening, and continuum slope variations indicates that the median quasar spectrum has much weaker EUV $(\lambda \lesssim 1100 \AA)$ relative flux than found in any previous studies. We attribute the difference in large part to the tendency of UV spectroscopic studies to preferentially select the bluest UV quasars, relative to optical brightness.

The catalogue matching results highlight the following points. There is a small but significant offset in the GALEX versus SDSS astrometry that can be reconciled with small rotational offsets. The standard deviation of the object matches is about 0.7 arcsec, increasing with distance from a GALEX tile centre, and NUV magnitude. Catalogued GALEX detections obscure a region around them that extends at least 10 arcsec in radius, and can extend up to 30 arcsec in radius. This causes an estimated 10 per cent of otherwise detectable quasars to be missing from the GALEX catalogue. About 95 percent of true matches are found at separations less than 3 arcsec, but true matches can be found out to separations as wide as 7 arcsec. NUV and FUV magnitude limits, which are not given in the GALEX catalogue, can be estimated with simple linear relationships with effective exposure time. All of the covered SDSS quasars therefore have UV detection limits. Nearby SDSS neighbours of SDSS quasars affect the FUV - NUV colours of the quasars out to separations of about 10 arcsec, typically making them appear redder. These results were used to construct samples of quasars that are unambiguously detected or undetected in the GALEX photometry.

The UV versus optical colours of the quasars exhibit a red tail present at most redshifts that becomes more pronounced when non-detections are accounted for. Leveraging the nearly complete detection rate in the SDSS $u$ band, and applying survival statistics, the median FUV - NUV versus redshift trend was constructed for quasars up to $z \approx 2.8$. The colour trend corrected for nondetections becomes much redder at $z \gtrsim 0.5$ than its detected-only counterpart. The colour-redshift curve represents quasar spectra from approximately 400 to $2300 \AA$.

A template quasar spectral model passed through the GALEX bandpasses shows that absorption by the IGM is not in itself sufficient to explain the very red UV colours. The best-fitting model is consistent with no intrinsic reddening, a FUV continuum slope of $\alpha_{\nu, \text { FUV }}=-0.34$, and an EUV continuum slope of $\alpha_{v \text {, EUV }}=$ -2.90 . The reddest EUV slope found in previous composite spectral studies is about -2.0. Analysis of the GALEX colours of quasars in UV spectral studies shows that they tend to be substantially bluer than the SDSS sample used here. This selection bias is likely the primary cause of the differences with the EUV slope we find here.

The very soft EUV region of the best-fitting template can be accommodated by more detailed models of emission from accretion discs around supermassive black holes (Hubeny et al. 2000; Blaes 2004), in particular when the accretion rate is relatively low for a given black hole mass. Models that include disc winds or some other mass-loss are also more likely to soften the EUV flux than standard disc models. EUV colours of quasars in different subsamples, separated for example by black hole mass, Eddington ratio, luminosity, radio and $\mathrm{X}$-ray properties, and emission line properties should provide stronger tests of the quasar emission mechanism.

Finally, it should be mentioned that a byproduct of this study is a rather large candidate list of He II reionization probes with GALEX detections (Syphers et al. 2009; Worseck \& Prochaska
2011). Using the same matching and selection criteria as in Section 2.3, we find over $900 z \geq 2.7$ quasars with highly reliable NUV detections, and almost 300 with FUV detections. It is beyond the scope of the current study to analyse the list in detail, but it will be important to identify those rare quasar sightlines that may be observable with $H S T$ spectrographs while they remain available.

\section{ACKNOWLEDGEMENTS}

This project was supported in part by National Aeronautics and Space Administration grant NNX15AI72G. DEVB gratefully acknowledges support from a Saint Vincent College faculty research grant. Based on observations made with the Galaxy Evolution Explorer. The Galaxy Evolution Explorer was operated for the National Aeronautics and Space Administration by the California Institute of Technology under contract NAS5-98034. Some of the data described here may be obtained from the Mikulski Archive for Space Telescopes at https://dx.doi.org/10.17909/T9H59D. Support for the Mikulski Archive for Space Telescopes for non-Hubble Space Telescope data is provided by the National Aeronautics and Space Administration Office of Space Science via grant NNX13AC07G and by other grants and contracts. The authors thank the anonymous referee for helpful comments. The catalogue is being hosted on VizieR, which is operated by the Centre de Donnees astronomiques de Strasbourg (CDS; Ochsenbein, Bauer \& Marcout 2000). The Sloan Digital Sky Survey web site is www.sdss.org.

\section{REFERENCES}

Abolfathi B. et al., 2018, ApJS, 235, 42

Allen J. T., Hewett P. C., Maddox N., Richards G. T., Belokurov V., 2011, MNRAS, 410, 860

Barger A. J., Cowie L. L., 2010, ApJ, 718, 1235

Bianchi L., Shiao B., Thilker D., 2017, ApJS, 230, 24

Binette L. et al., 2005, ApJ, 631, 661

Blaes O., 2004, in Richards G. T., Hall P. B., eds, ASP Conf. Ser. Vol. 211, AGN Physics with the Sloan Digital Sky Survey. Astron. Soc. Pac., San Francisco, p. 121

Budavári T. et al., 2009, ApJ, 694, 1281

Capellupo D. M., Netzer H., Lira P., Trakhtenbrot B., Mejía-Restrepo J., 2015, MNRAS, 446, 3427

Cartledge S. I. B. et al., 2005, ApJ, 630, 355

Chen Y. Y., Hollander M., Langberg N. A., 1982, J. Am. Stat. Assoc., 77, 141

Crenshaw D. M., Kraemer S. B., George I. M., 2003, ARA\&A, 41, 117

Czerny B., Li J., Loska Z., Szczerba R., 2004, MNRAS, 348, L54

Davis S. W., Woo J.-H., Blaes O. M., 2007, ApJ, 668, 682

de Diego J. A., Binette L., Ogle P., Andersen A. C., Haro-Corzo S., Wold M., 2007, A\&A, 467, L7

Deharveng J.-M., Milliard B., Péroux C., Small T., 2019, A\&A, 623, A149

Feigelson E. D., Nelson P. I., 1985, ApJ, 293, 192

Francis P. J., Hewett P. C., Foltz C. B., Chaffee F. H., Weymann R. J., Morris S. L., 1991, ApJ, 373, 465

Fynbo J. P. U., Krogager J.-K., Venemans B., Noterdaeme P., Vestergaard M., Møller P., Ledoux C., Geier S., 2013, ApJS, 204, 6

Gaskell C. M., Goosmann R. W., Antonucci R. R. J., Whysong D. H., 2004, ApJ, 616, 147

Haardt F., Madau P., 2012, ApJ, 746, 125

Hammer D., Hornschemeier A. E., Mobasher B., Miller N., Smith R., Arnouts S., Milliard B., Jenkins L., 2010, ApJS, 190, 43

Haro-Corzo S. A. R., Binette L., Krongold Y., Benitez E., Humphrey A., Nicastro F., Rodríguez-Martínez M., 2007, ApJ, 662, 145

Heintz K. E. et al., 2016, A\&A, 595, A13

Hjorth J., Vreeswijk P. M., Gall C., Watson D., 2013, ApJ, 768, 173

Hopkins P. F. et al., 2004, AJ, 128, 1112 
Hubeny I., Agol E., Blaes O., Krolik J. H., 2000, ApJ, 533, 710

Hubeny I., Blaes O., Krolik J. H., Agol E., 2001, ApJ, 559, 680

Inoue A. K., Shimizu I., Iwata I., Tanaka M., 2014, MNRAS, 442, 1805

Jiang P. et al., 2013, AJ, 145, 157

Kalambet Y., Kozmin Y., Mikhailova K., Nagaev I., Tikhonov P., 2011, J. Chemometr., 25, 352

Kaplan E. L., Meier P., 1958, J. Am. Stat. Assoc., 53, 457

Kazanas D., 2019, Galaxies, 7, 13

Khan H. R., Shaw J. E. H., 2016, J. Stat. Theory Pract., 10, 7

Kolykhalov P. I., Sunyaev R. A., 1984, Adv. Space Res., 3, 249

Koratkar A. P., 1999, in Gaskell C. M., Brandt W. N., Dietrich M., DultzinHacyan D., Eracleous M., eds, ASP Conf. Ser. Vol. 175, Structure and Kinematics of Quasar Broad Line Regions. Astron. Soc. Pac., San Francisco, p. 131

Koratkar A., Blaes O., 1999, PASP, 111, 1

Krawczyk C. M., Richards G. T., Mehta S. S., Vogeley M. S., Gallagher S. C., Leighly K. M., Ross N. P., Schneider D. P., 2013, ApJS, 206, 4

Krawczyk C. M., Richards G. T., Gallagher S. C., Leighly K. M., Hewett P. C., Ross N. P., Hall P. B., 2015, AJ, 149, 203

Krogager J.-K. et al., 2016, ApJ, 832, 49

Laor A., 1990, MNRAS, 246, 369

Laor A., Davis S. W., 2014, MNRAS, 438, 3024

Laor A., Netzer H., 1989, MNRAS, 238, 897

Leighly K. M., Terndrup D. M., Baron E., Lucy A. B., Dietrich M., Gallagher S. C., 2014, ApJ, 788, 123

Lupton R. H., Gunn J. E., Szalay A. S., 1999, AJ, 118, 1406

Lusso E., Worseck G., Hennawi J. F., Prochaska J. X., Vignali C., Stern J., O’Meara J. M., 2015, MNRAS, 449, 4204

Maddox N., Hewett P. C., Warren S. J., Croom S. M., 2008, MNRAS, 386, 1605

Martin D. C. et al., 2005, ApJ, 619, L1

Mathews W. G., Ferland G. J., 1987, ApJ, 323, 456

Moloney J., Shull J. M., 2014, ApJ, 793, 100

Morrissey P. et al., 2007, ApJS, 173, 682

Ochsenbein F., Bauer P., Marcout J., 2000, A\&AS, 143, 23

Pâris I. et al., 2017, A\&A, 597, A79

Pâris I. et al., 2018, A\&A, 613, A51

Peek J. E. G., Graves G. J., 2010, ApJ, 719, 415

Peek J. E. G., Schiminovich D., 2013, ApJ, 771, 68

Pier J. R., Munn J. A., Hindsley R. B., Hennessy G. S., Kent S. M., Lupton R. H., Ivezić Ž., 2003, AJ, 125, 1559

Planck Collaboration XI, 2014, A\&A, 571, 11

Rai R. K., Rastogi S., 2010, MNRAS, 401, 2722

Ribaudo J., Lehner N., Howk J. C., 2011, ApJ, 736, 42

Richards G. T. et al., 2002, AJ, 123, 2945

Richards G. T. et al., 2003, AJ, 126, 1131

Ross R. R., Fabian A. C., Mineshige S., 1992, MNRAS, 258, 189

Schlafly E. F., Finkbeiner D. P., 2011, ApJ, 737, 103

Schlafly E. F., Finkbeiner D. P., Schlegel D. J., Jurić M., Ivezić Ž., Gibson

R. R., Knapp G. R., Weaver B. A., 2010, ApJ, 725, 1175

Schlegel D. J., Finkbeiner D. P., Davis M., 1998, ApJ, 500, 525 (SFD)

Scott A. E., Stewart G. C., 2014, MNRAS, 438, 2253

Scott J. E., Kriss G. A., Brotherton M., Green R. F., Hutchings J., Shull J. M., Zheng W., 2004, ApJ, 615, 135
Selsing J., Fynbo J. P. U., Christensen L., Krogager J.-K., 2016, A\&A, 585, A87

Shang Z. et al., 2005, ApJ, 619, 41

Shankar F. et al., 2016, ApJ, 818, L1

Shields G. A., 1978, Nature, 272, 706

Shull J. M., Tumlinson J., Giroux M. L., Kriss G. A., Reimers D., 2004, ApJ, 600, 570

Shull J. M., Stevans M., Danforth C. W., 2012, ApJ, 752, 162

Sincell M. W., Krolik J. H., 1998, ApJ, 496, 737

Slone O., Netzer H., 2012, MNRAS, 426, 656

Stevans M. L., Shull J. M., Danforth C. W., Tilton E. M., 2014, ApJ, 794, 75

Sun W.-H., Malkan M. A., 1989, ApJ, 346, 68

Sun M., Jiang B. W., Zhao H., Gao J., Gao S., Jian M., Yuan H., 2018, ApJ, 861,153

Syphers D., Anderson S. F., Zheng W., Haggard D., Meiksin A., Schneider D. P., York D. G., 2009, ApJS, 185, 20

Telfer R. C., Zheng W., Kriss G. A., Davidsen A. F., 2002, ApJ, 565, 773

Tilton E. M., Stevans M. L., Shull J. M., Danforth C. W., 2016, ApJ, 817, 56

Trammell G. B., Vanden Berk D. E., Schneider D. P., Richards G. T., Hall P. B., Anderson S. F., Brinkmann J., 2007, AJ, 133, 1780

Upton Sanderbeck P. R., McQuinn M., D’Aloisio A., Werk J. K., 2018, ApJ, 869,159

Vanden Berk D. E. et al., 2001, AJ, 122, 549

Weingartner J. C., Draine B. T., 2001, ApJ, 548, 296

Wethers C. F. et al., 2018, MNRAS, 475, 3682

Wolf C., 2014, MNRAS, 445, 4252

Worseck G., Prochaska J. X., 2011, ApJ, 728, 23

Xie X., Shao Z., Shen S., Liu H., Li L., 2016, ApJ, 824, 38

York D. G. et al., 2000, AJ, 120, 1579

Zafar T. et al., 2015, A\&A, 584, A100

Zheng W., Kriss G. A., Telfer R. C., Grimes J. P., Davidsen A. F., 1997, ApJ, 475, 469

Zhong M., Hess K. R., 2009, Collection of Biostatistics Research Archive, 66,1

\section{SUPPORTING INFORMATION}

Supplementary data are available at MNRAS online.

\section{ReadMe.dat staa411}

Please note: Oxford University Press is not responsible for the content or functionality of any supporting materials supplied by the authors. Any queries (other than missing material) should be directed to the corresponding author for the article.

This paper has been typeset from a $\mathrm{T}_{\mathrm{E}} \mathrm{X} / \mathrm{L} \mathrm{T}_{\mathrm{E}} \mathrm{X}$ file prepared by the author. 